\title{
Article \\ Practical Synthesis of Phosphinic Dipeptides by Tandem Esterification of Aminophosphinic and Acrylic Acids under Silylating Conditions
}

\author{
Paraskevi Kokkala ${ }^{1,+}$, Kostas Voreakos ${ }^{1,+}{ }^{,}$Angelos Lelis ${ }^{1}$, Konstantinos Patiniotis ${ }^{1}$, Nikolaos Skoulikas ${ }^{1}$ (D), \\ Laurent Devel $^{2}$ (D) Angeliki Ziotopoulou ${ }^{1}$, Eleni Kaloumenou ${ }^{1}$ and Dimitris Georgiadis ${ }^{1, *(D)}$
}

1 Laboratory of Organic Chemistry, Department of Chemistry, National and Kapodistrian University of Athens, Panepistimiopolis, Zografou, 15784 Athens, Greece; pkokkala@chem.uoa.gr (P.K.); chemnite@chem.uoa.gr (K.V.); angeloslelis@gmail.com (A.L.); konstantinp@chem.uoa.gr (K.P.); nikolas.skoulikas@gmail.com (N.S.); kelisia1995@hotmail.com (A.Z.); lenakal17@gmail.com (E.K.)

2 Département Médicaments et Technologies pour la Santé (DMTS), CEA, INRAE, SIMoS, Université Paris-Saclay, 91191 Gif-sur-Yvette, France; laurent.devel@cea.fr

* Correspondence: dgeorgia@chem.uoa.gr; Tel.: +30-2107274903

+ These authors contributed equally to this work.

\section{check for} updates

Citation: Kokkala, P.; Voreakos, K.; Lelis, A.; Patiniotis, K.; Skoulikas, N.; Devel, L.; Ziotopoulou, A.; Kaloumenou, E.; Georgiadis, D. Practical Synthesis of Phosphinic Dipeptides by Tandem Esterification of Aminophosphinic and Acrylic Acids under Silylating Conditions. Molecules 2022, 27, 1242. https:// doi.org/10.3390/molecules27041242

Academic Editor: György Keglevich

Received: 21 January 2022

Accepted: 10 February 2022

Published: 12 February 2022

Publisher's Note: MDPI stays neutral with regard to jurisdictional claims in published maps and institutional affiliations.

Copyright: (C) 2022 by the authors. Licensee MDPI, Basel, Switzerland. This article is an open access article distributed under the terms and conditions of the Creative Commons Attribution (CC BY) license (https:// creativecommons.org/licenses/by/ $4.0 /)$.

\begin{abstract}
In this report, a synthetic protocol for the preparation of phosphinic dipeptides of type 5 is presented. These compounds serve as valuable building blocks for the development of highly potent phosphinopeptidic inhibitors of medicinally relevant Zn-metalloproteases and aspartyl proteases. The proposed method is based on the tandem esterification of $\alpha$-aminophosphinic and acrylic acids under silylating conditions in order to subsequently participate in a $P$-Michael reaction. The scope of the transformation was investigated by using a diverse set of readily available acrylic acids and $(R)-\alpha-$ aminophosphinic acids, and high yields were achieved in all cases. In most examples reported herein, the isolation of biologically relevant $(R, S)$-diastereoisomers became possible by simple crystallization from the crude products, thus enhancing the operational simplicity of the proposed method. Finally, functional groups corresponding to acidic or basic natural amino acids are also compatible with the reaction conditions. Based on the above, we expect that the practicality of the proposed protocol will facilitate the discovery of pharmacologically useful bioactive phosphinic peptides.
\end{abstract}

Keywords: phosphinic; pseudopeptides; P-Michael; silylation; hexamethyldisilazane; inhibitors; Zn-metalloproteases; acrylic acids; aminophosphinic; organophosphorus

\section{Introduction}

Among the plethora of bioactive scaffolds with numerous applications in drug discovery, phosphinic peptides undoubtedly hold a prominent position [1-9]. By acting as transition state analogues of natural peptides during proteolysis, phosphinic peptides have provided a large number of potent inhibitors of zinc and aspartyl proteases over previous decades $[2,5,6]$. In addition, their propensity to bind tightly to their target has fueled efforts towards the development of enzymatic probes for imaging applications [10-14]. Their synthesis is typically based on building block strategies where side chains of the phosphinopeptidic scaffold are either preinstalled or introduced at a later stage of the synthetic plan by post-modification protocols [3,4]. Therefore, it becomes evident that synthetic methodologies aiming to the fast and reliable production of phosphinic dipeptide building blocks lie at the heart of every medicinal project involving this privileged class of bioactive compounds.

There are two possible synthetic approaches towards a phosphinodipeptidic building block: the "NP + C approach", which relies on P-C bond-forming reactions between aminophosphinic derivatives and carbon electrophiles, and the less-commonly 
used "N + PC approach" which involves Mannich-type or amidoalkylation reactions with 3-phosphinoyl propionates of type I (Scheme 1) $[3,15,16]$. The preference of synthetic chemists for the NP $+\mathrm{C}$ approach mainly stems from the fact that optically pure $\alpha$-aminophosphinic acids can be accessed by well-established methods [17-19], thus allowing stereochemical efficiency and scalability. In particular, diacids of type $\mathbf{5}$ (Scheme 2) have been successfully employed as starting materials in the discovery of potent and selective inhibitors of a wide range of proteases, such as ER aminopeptidases [20], neutral aminopeptidase (APN) [21,22], matrix metalloproteinases (MMPs) [23], neprilysin [22], angiotensinconverting enzymes (ACE) 1 and 2 [24,25], endothelin-converting enzyme (ECE) [24] and many more [22,26-28]. In most cases, such compounds are produced by the initial conversion of $\alpha$-aminophosphinic acids of type 1 to the respective bis(trimethylsilyl)phosphonites $\mathbf{1}^{\prime}$, followed by the addition of $\alpha$-substituted acrylates (2) to the resulting $P$-nucleophiles (Scheme 2) $[3,29,30]$. Since the reaction affords carboxylic esters (4), an additional cleavage step is required to furnish the final compounds of type $5[20,23,31]$. Interestingly, a more direct, protecting-group-free approach which involves the use of acrylic acids of type 3 as electrophiles (Scheme 2) has to our knowledge never been reported in the literature, probably because acrylic acids are intuitively excluded when different electrophile alternatives are considered.

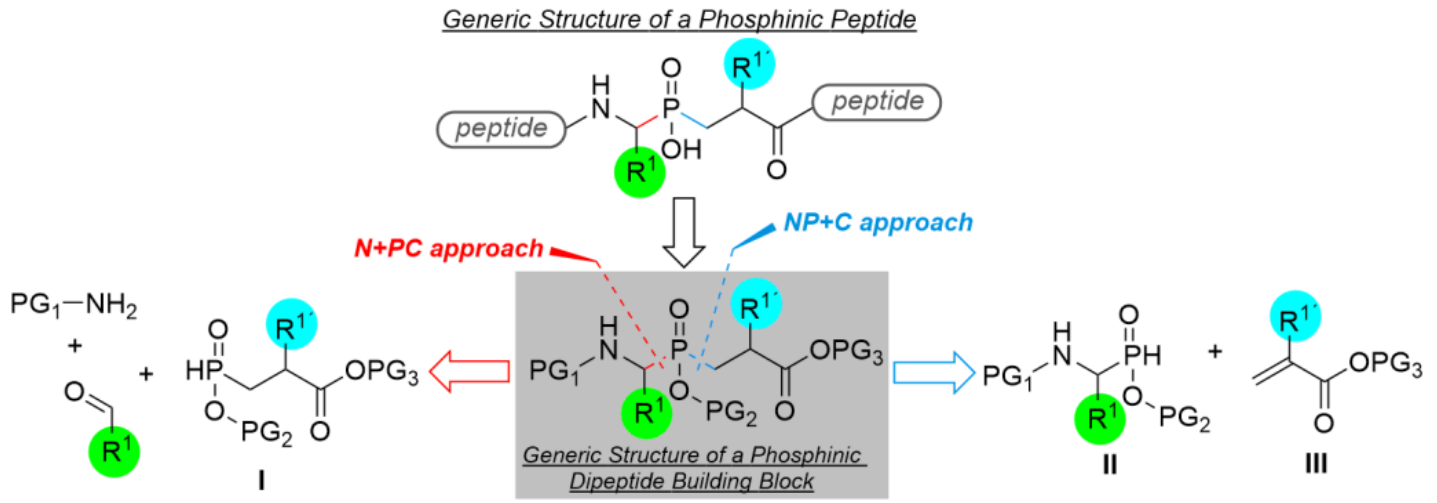

Scheme 1. General retrosynthetic approaches for the synthesis of phosphinic peptides.

Since $P$-Michael additions using phosphinic acids are mainly performed under silylating conditions, we assumed that acrylic acids could also be silylated towards the corresponding esters and react with the P-nucleophiles generated in situ (Scheme 2). By extending our literature search to different types of phosphinic acids, only scarce examples of relevant reactions were identified, either leading to moderate yields [32] or employing highly reactive electrophiles [33]. Given this striking gap in the literature, we decided to investigate the feasibility of such a transformation, aiming not only to a shorter synthetic route for the preparation of phosphinic building blocks of type 5, but also to increased simplicity and scalability. Moreover, there are several examples in the literature where the biologically relevant, less soluble $(R, S)$-diastereoisomer of Cbz-protected diacids 5 can be easily separated from the $(R, R)$-isomer by crystallization $[20,23,25,31,34]$, which implies that the proposed protocol may have the potential to lead directly to optically pure $(R, S)$-phosphinic dipeptides without the need of chromatographic separation. To this respect, in the present study we explore the synthesis of phosphinopeptidic building blocks of type $\mathbf{5}$ by tandem activation of $\alpha$-aminophosphinic acids (1) and temporary esterification of acrylic acids (3) in a $P$-Michael reaction under silylating conditions, aiming to a protocol that will be characterized by high overall practicality and synthetic convenience. 


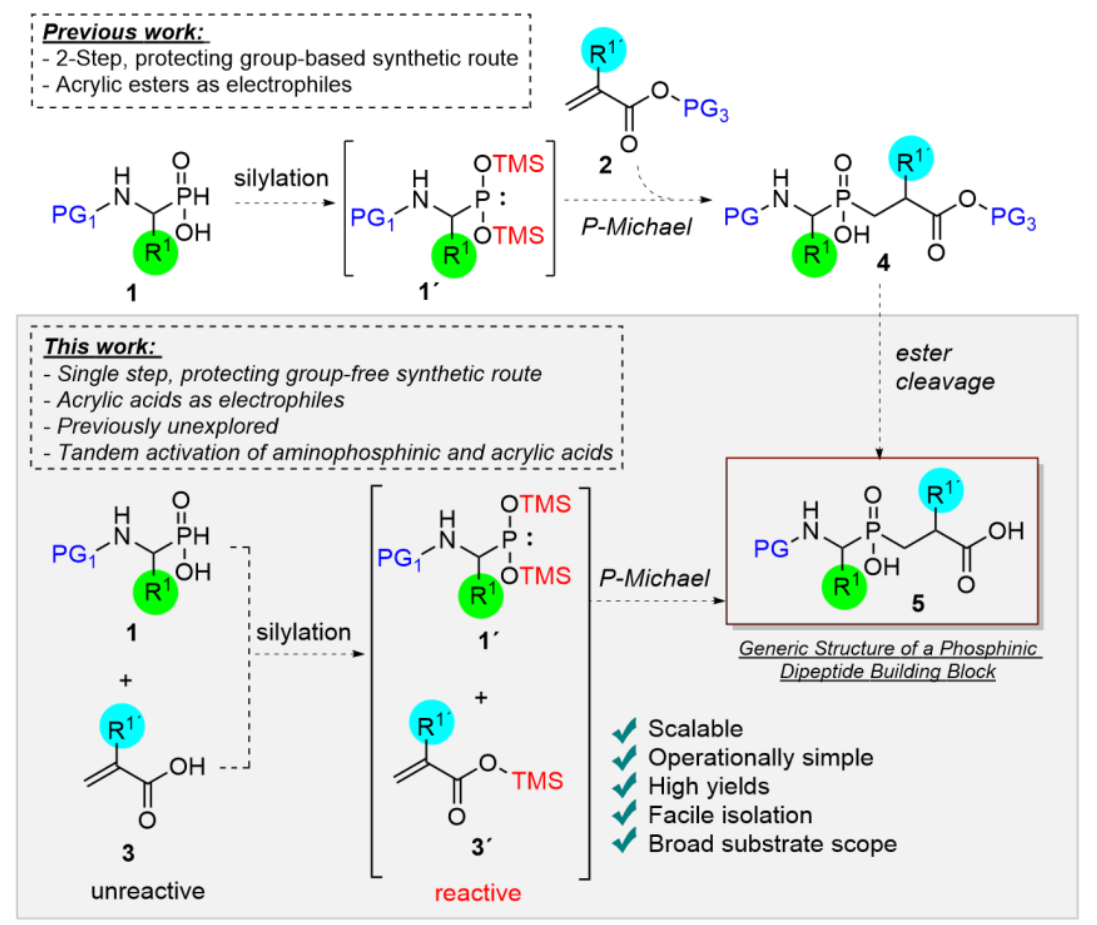

Scheme 2. Schematic representation of previously described protocol towards the synthesis of building blocks of type 5 and proposed protocol in this study.

\section{Results and Discussion}

\subsection{Preparation of Acrylic Acids of Type 3}

Minimization of chromatographic purification steps in a synthetic plan is a crucial parameter that needs to be taken into account for assessing the overall cost efficiency of a preparation, especially when upscaling is required. In this regard, as it was mentioned in the introduction part, simple $\alpha$-aminophosphinic acids of type 1 can be accessed in multigram quantities and optically pure form by following the classical method of Baylis et al. [19]. On the other hand, $\alpha$-substituted acrylic acids of type 3 can be accessed by a 3-step procedure starting from diethyl malonate and simple alkyl halides, as described in Scheme 3.

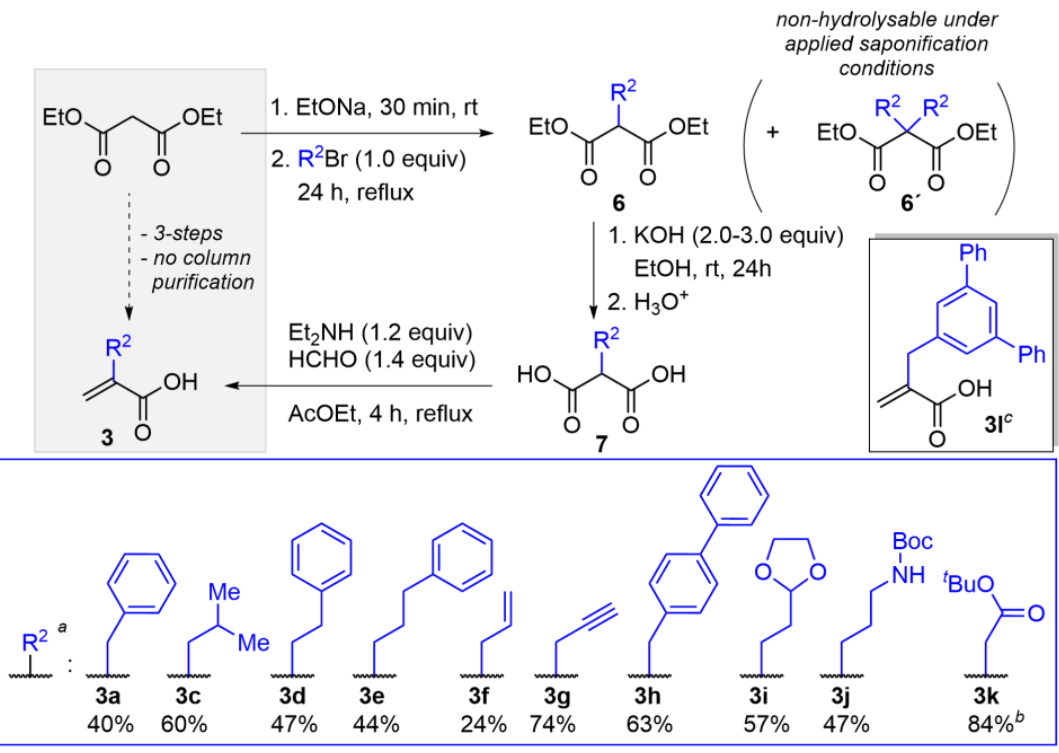

Scheme 3. Synthesis of acrylic acids $3 \mathbf{a}$ and $\mathbf{3} \mathbf{c}-\mathbf{k}$ used in this study (see Supplementary Material). ${ }^{a}$ Isolated yields are given. ${ }^{b} \mathrm{NaH}$ was used for the alkylation step [35]. ${ }^{c}$ Prepared according to ref. [20]. 
In the first step, the alkylation of diethyl malonate leads to malonic derivatives of type 6 , generally contaminated with small amounts of dialkylated by-products $\left(6^{\prime}\right)$ and unreacted materials. The removal of dialkylated derivatives $\mathbf{6}^{\prime}$ becomes possible at the next step by taking advantage of their low reactivity towards hydrolysis due to increased steric hindrance. This allows the facile isolation of the target malonic acids of type 7 by simple aqueous workup. At the final step of the procedure, a Doebner-Knoevenagel condensation with formaldehyde takes place, leading to adequately pure target acrylic acids of type 3 without the need for chromatographic purification and overall yields ranging from 24 to $84 \%$ for 3 steps. The structure of acrylic acids of type 3 prepared by the above method is shown in Scheme 3.

\subsection{Optimization}

Next, we proceeded to the evaluation of our proposed method by employing the Cbz-protected aminophosphinic acid 1a and acrylic acid 3a as starting materials in our initial experiments (Table 1).

Table 1. Optimization of reaction conditions.

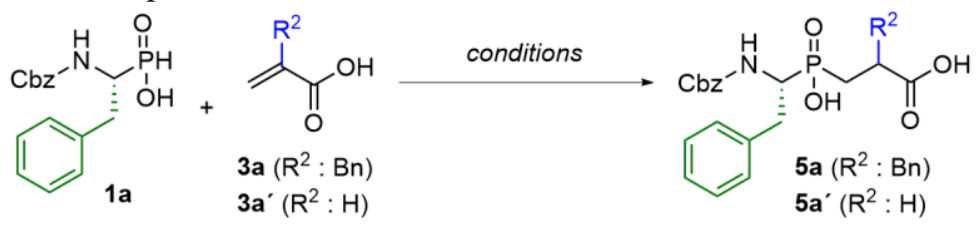

\begin{tabular}{|c|c|c|c|}
\hline Entry & Acrylic Acid (Equiv) & Reaction Conditions $^{1}$ & $\begin{array}{l}\text { Conversion of } 1 a, \% \\
\text { (yield to } 5 a, \%)^{2}\end{array}$ \\
\hline 1 & $3 a(1.3)$ & BSA ( 4 equiv), $\mathrm{CH}_{2} \mathrm{Cl}_{2}, \mathrm{rt}, 48 \mathrm{~h}$ & $53(41)$ \\
\hline 2 & $3 \mathbf{a}^{\prime}(1.3)$ & BSA ( 4 equiv), $\mathrm{CH}_{2} \mathrm{Cl}_{2}, \mathrm{rt}, 48 \mathrm{~h}$ & $90(89)^{3}$ \\
\hline 3 & $3 a(1.3)$ & $\begin{array}{c}\mathrm{TMSCl}\left(7 \text { equiv) },{ }^{i} \operatorname{Pr}_{2} \mathrm{EtN} \text { (7 }\right. \\
\text { equiv), } \mathrm{CH}_{2} \mathrm{Cl}_{2}, \mathrm{rt}, 48 \mathrm{~h}\end{array}$ & $86(81)$ \\
\hline 4 & $3 \mathbf{a}(1.2)$ & $\operatorname{HMDS}\left(6\right.$ equiv), $100^{\circ} \mathrm{C}, 2 \mathrm{~h}$ & $100(99)$ \\
\hline 5 & $3 a(1.2)$ & HMDS ( 6 equiv), $70^{\circ} \mathrm{C}, 2 \mathrm{~h}$ & $72(70)$ \\
\hline
\end{tabular}

${ }^{1}$ All reactions were performed in a $0.3 \mathrm{mmol}$ scale of $1 \mathrm{a}$ under inert conditions. ${ }^{2}$ Conversions and yields were determined by integration of the ${ }^{31} \mathrm{P}$-NMR spectra of crude products. ${ }^{3}$ Conversion to $5 \mathrm{a}^{\prime}$.

Based on previous reports [32,33], we first tested the efficiency of BSA [N,O-bis(trimethy lsilyl)acetamide] in promoting the $P$-Michael reaction between $1 \mathbf{a}$ and $3 \mathbf{a}$. The reaction of a mixture of $\mathbf{1 a}$ (1.0 equiv), 3a (1.2 equiv) and BSA (4 equiv) for $48 \mathrm{~h}$ led to just a 53\% conversion of the starting material and $41 \%$ formation of target compound $5 \mathbf{a}$, as it was judged by the ${ }^{31} \mathrm{P}-\mathrm{NMR}$ spectrum of the crude mixture. This was in accordance with the low efficiency observed in the literature for a similar reaction [32]. When unsubstituted acrylic acid (3a') was used, the conversion to product 5a' increased to $90 \%$ under the same conditions, which implies that steric effects due to the presence of a substituent in 2-position of acrylic acids are partially responsible for the low reactivity of studied electrophiles. TMSCl as silylating agent in the presence of Hünig's base proved more efficient for the formation of product $5 \mathbf{a}(82 \%)$, based on ${ }^{31} \mathrm{P}-\mathrm{NMR}$, leaving only $13 \%$ of unreacted 1a. Despite the improved reaction profile, long reaction times associated with these conditions prompted us to explore hexamethyldisilazane (HMDS) as an alternative, which is known to cause silylation of acidic functionalities upon heating [29]. Indeed, heating of a mixture of 1a, 3a and 6 equiv of HMDS to $100{ }^{\circ} \mathrm{C}$ during $2 \mathrm{~h}$ under inert conditions led to quantitative formation of the desired product 5a. Lowering the temperature to $70{ }^{\circ} \mathrm{C}$ had a negative effect to the conversion of phosphinic acid 1a, presumably due to incomplete activation of the reactants. 


\subsection{Substrate Scope}

After identifying the optimal conditions for the $P$-Michael addition of $\alpha$-aminophoshinic acids to acrylic acids through their tandem esterification by silylation, we proceeded to the application of our protocol in a wide range of substrates, as shown in Scheme 4.

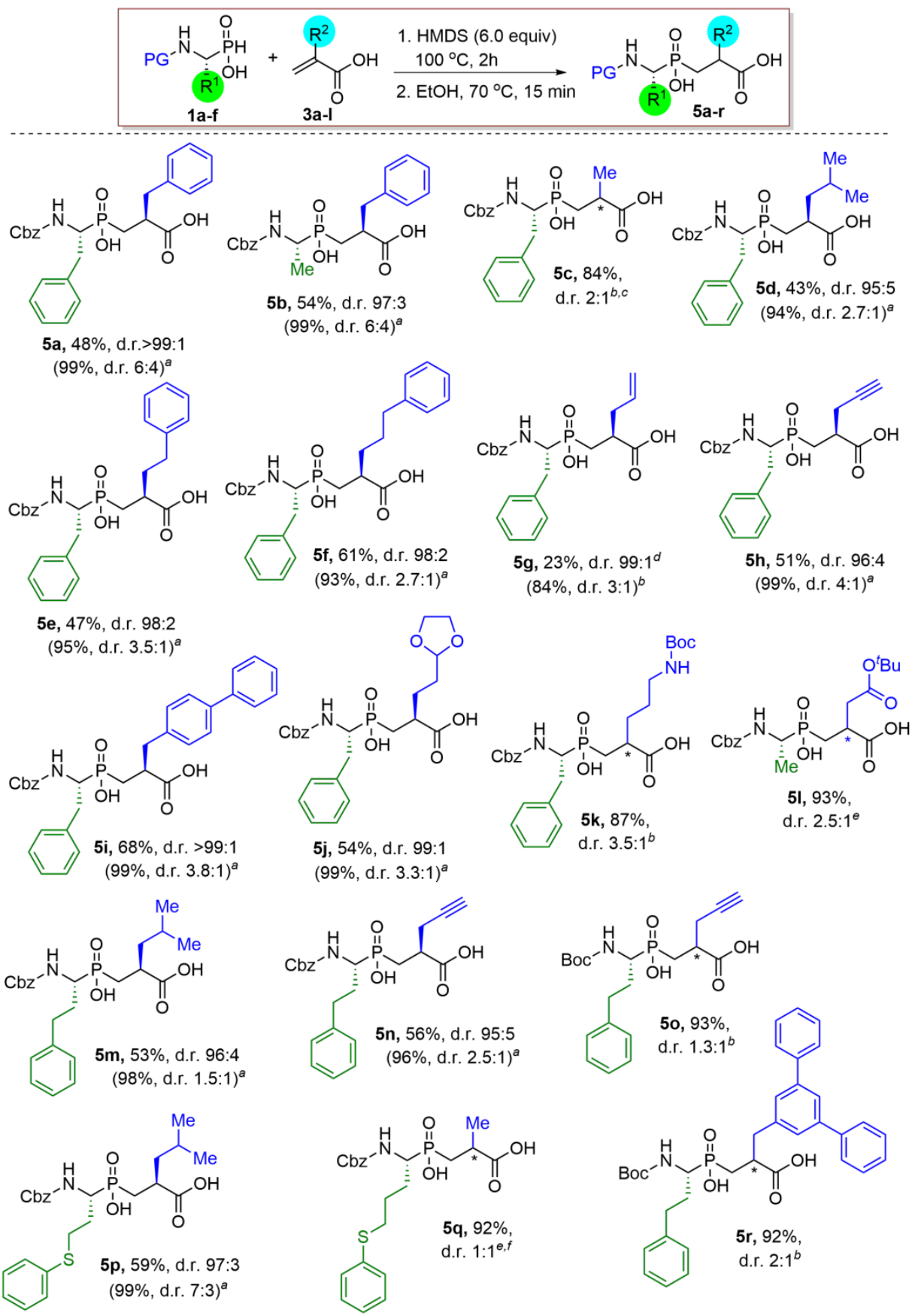

Scheme 4. Substrate scope for the proposed $P$-Michael reaction of aminophosphinic (1) and acrylic (3) acids by tandem esterification under silylating conditions. Isolated yields are provided in all examples. ${ }^{a}$ outside parentheses: isolated yields to 5 and d.r. values after separation of $(R, S)$-isomer by crystallization; inside parentheses: conversions to 5 and d.r. values from crude products, as estimated by integration of their ${ }^{31} \mathrm{P}$-NMR spectra; ${ }^{b}$ isolated yield after column purification; ${ }^{c}$ d.r. was estimated by integration of ${ }^{31} \mathrm{P}-\mathrm{NMR}$ spectrum in $\mathrm{CD}_{3} \mathrm{OD} ;{ }^{d}$ recrystallized by $\mathrm{CHCl}_{3} ;{ }^{e}$ isolated yield after treatment with $\mathrm{Et}_{2} \mathrm{O} ;{ }^{f}$ d.r. was estimated by integration of the ${ }^{31} \mathrm{C}-\mathrm{NMR}$ spectrum of isolated product $\mathbf{5 q}$. 
Since $(R)$ - $\alpha$-aminophosphinic acids were used, a mixture of two diastereoisomers $[(R, S)$ and $(R, R)]$ was obtained in all cases. However, as mentioned above, the $(R, S)$ diastereoisomer of Cbz-protected diacids of type 5 has a higher tendency to crystallize compared to $(R, R)$ isomers. This was observed in 12 out of 16 examples of Cbz-protected diacids prepared in this study, allowing the isolation of target compounds of type 5 with high diastereoisomeric purity, as determined by ${ }^{31} \mathrm{P}-\mathrm{NMR}$, without the need of chromatographic purification. Considering that starting materials were also obtained without column chromatography, it becomes evident that the proposed protocol is characterized by high practicality and scalability. Boc-protected $\alpha$-aminophosphinic acids are also compatible with this protocol leading to compounds 50 and $5 \mathbf{r}$ in high yields. However, in these cases the presence of Boc group hampered diastereoisomeric separation. Furthermore, acrylic acids with functional groups corresponding to the side chains of acidic (5l) or basic (5k) aminoacids were also compatible with the proposed method.

\section{Materials and Methods}

\subsection{General Information}

All P-Michael reactions were carried out under argon atmosphere. All solvents were used without further purification. Reagents were purchased at the highest commercial quality from Aldrich (St. Louis, MI, USA), Acros (Belgium), Fluka and Fluorochem and were used without further purification. Reactions were monitored by thin-layer chromatography (TLC) carried out on $0.25 \mathrm{~mm}$ silica gel plates (E. Merck silica gel $60 \mathrm{~F}_{254}$ ) and components were visualized by the following methods: UV light absorbance, and/or charring after spraying with a solution of $\mathrm{NH}_{4} \mathrm{HSO}_{4}$ or an aqueous solution of cerium molybdate $/ \mathrm{H}_{2} \mathrm{SO}_{4}$ ("Blue Stain"), or an aqueous solution of $\mathrm{KMnO}_{4}$ and heating. Purification of compounds by column chromatography was carried out on silica gel (Merck (Darmstadt, Germany), 70-230 mesh) and the indicated solvents. Melting points (measured on an Electrothermal apparatus) are uncorrected. ${ }^{1} \mathrm{H},{ }^{13} \mathrm{C}$ and ${ }^{31} \mathrm{P}$ NMR spectra were recorded on a Varian $200 \mathrm{MHz}$ Mercury spectrometer and on a Bruker Avance $\mathrm{Neo} 400 \mathrm{MHz}$ at $25{ }^{\circ} \mathrm{C} .{ }^{1} \mathrm{H}$ and ${ }^{13} \mathrm{C}$ spectra are referenced according to the residual peak of the solvent based on literature data [36]. ${ }^{31} \mathrm{P}$ NMR chemical shifts are reported in ppm downfield from $85 \% \mathrm{H}_{3} \mathrm{PO}_{4}$ (external standard). ${ }^{13} \mathrm{C}$ and ${ }^{31} \mathrm{P}$ NMR spectra are fully proton-decoupled. Phosphinic diacids of type 5 tend to aggregate, causing peak broadening in the NMR spectra. In all products of type 5, small signals observed upfield from the main peaks corresponded to rotamers observed in this type of compound, as it has been reported in the literature [37]. Signals marked with an asterisk $\left(^{*}\right)$ in the NMR assignment correspond to minor isomers. Optical rotation data were obtained in an Optical Activity instrument. High-resolution mass spectra were obtained on a Bruker Maxis Impact QTOF spectrometer or an AB Sciex 4600 Triple TOF mass spectrometer.

\subsection{General Procedure for the HMDS-Mediated P-C Bond-Forming Reaction between Phosphinic and Acrylic Acids}

Phosphinic acid of type $\mathbf{1}$ (1.0 equiv) and acrylic acid of type 3 (1.2 equiv) were added to a round-bottomed flask. Then, HMDS (6.0 equiv) was slowly added and the flask was firmly closed with a septum and thoroughly purged with argon. Then, the mixture was slowly heated to $100{ }^{\circ} \mathrm{C}$, ensuring that gas $\mathrm{NH}_{3}$ was released (by periodically piercing the septum with a needle, taking care to always maintain a positive pressure inside the flask). Production of gas $\mathrm{NH}_{3}$ starts at $\sim 60^{\circ} \mathrm{C}$ and was completed at $100{ }^{\circ} \mathrm{C}$. The mixture was heated at $100{ }^{\circ} \mathrm{C}$ during $2 \mathrm{~h}$ and then cooled at $70{ }^{\circ} \mathrm{C}$. At this temperature, $\mathrm{EtOH}$ $(0.2 \mathrm{~mL} / \mathrm{mmol}$ of HMDS) was slowly added under a gentle flow of argon, and stirring was continued for $15 \mathrm{~min}$. The resulting mixture was diluted with $\mathrm{HCl} 2 \mathrm{M}(15 \mathrm{~mL} / \mathrm{mmol}$ of $\mathbf{1})$ and AcOEt $(20 \mathrm{~mL} / \mathrm{mmol}$ of $\mathbf{1})$. Isolation of the final product of type $\mathbf{5}$ is described separately for each example given below.

(2S)-2-Benzyl-3-\{[(R)-1-(benzyloxycarbonylamino)-2-henylethyl](hydroxy)phosphoryl\}propanoic acid (5a): Phosphinic acid 1a (100 mg, $0.31 \mathrm{mmol})$ and acrylic acid 3a (61 mg, $0.37 \mathrm{mmol})$ were subjected to 
the general HMDS-mediated P-C bond-forming reaction protocol. After the addition of $\mathrm{HCl} 2$ $\mathrm{M}$ and AcOEt, a solid was precipitated that was collected by filtration. The solid product was recrystallized by AcOEt to afford compound 5a (d.r. >99:1) as a white solid in $48 \%$ yield (72 mg, $0.15 \mathrm{mmol})$. m.p. $190-195^{\circ} \mathrm{C}$; TLC $R_{\mathrm{f}}\left(\mathrm{CHCl}_{3} / \mathrm{MeOH} / \mathrm{AcOH}: 7 / 2 / 1\right)=0.58 .{ }^{1} \mathrm{H}$ NMR $(500 \mathrm{MHz}$, $\mathrm{d}_{6}$-DMSO + 4\%TFA) $\delta 7.68(\mathrm{~d}, J=9.5 \mathrm{~Hz}, 1 \mathrm{H}), 7.37-6.94(\mathrm{~m}, 15 \mathrm{H}), 4.95(\mathrm{~d}, J=13 \mathrm{~Hz}, 1 \mathrm{H}), 4.88(\mathrm{~d}$, $J=13 \mathrm{~Hz}, 1 \mathrm{H}), 3.14-2.84(\mathrm{~m}, 4 \mathrm{H}), 2.78-2.68(\mathrm{~m}, 1 \mathrm{H}), 2.10-1.92(\mathrm{~m}, 1 \mathrm{H}), 1.84-1.74(\mathrm{~m}, 1 \mathrm{H}) ;{ }^{13} \mathrm{C}$ NMR (50 MHz, $\mathrm{d}_{6}$-DMSO) $\delta 176.5\left(\mathrm{~d},{ }^{3} J_{\mathrm{PC}}=7.6 \mathrm{~Hz}\right), 156.1\left(\mathrm{~d},{ }^{3} \mathrm{~J}_{\mathrm{PC}}=3.8 \mathrm{~Hz}\right), 138.9,138.6,138.4$, 137.3, 129.2, 128.4, 127.7, 127.1, 126.4, 65.3, $52.7\left(\mathrm{~d},{ }^{1} J_{\mathrm{PC}}=104 \mathrm{~Hz}\right), 40.5,38.5,32.8,27.6\left(\mathrm{~d},{ }^{1} J_{\mathrm{PC}}\right.$ $=89 \mathrm{~Hz}) ;{ }^{31} \mathrm{P}$ NMR $\left(81 \mathrm{MHz}, \mathrm{d}_{6}\right.$-DMSO + 4\%TFA) $\delta 46.4 ; \mathrm{HRMS}(\mathrm{m} / z):[\mathrm{M}+\mathrm{Na}]^{+}$calcd. for $\mathrm{C}_{26} \mathrm{H}_{28} \mathrm{NNaO}_{6} \mathrm{P}^{+}, 504.1547$ found, 504.1565.

(2S)-2-Benzyl-3-\{[(R)-1-(benzyloxycarbonylamino)ethyl](hydroxy)phosphoryl\}propanoic acid (5b): Phosphinic acid $\mathbf{1 b}(100 \mathrm{mg}, 0.41 \mathrm{mmol})$ and acrylic acid $3 \mathbf{a}(80 \mathrm{mg}, 0.49 \mathrm{mmol})$ were subjected to the general HMDS-mediated P-C bond-forming reaction protocol. After the addition of $\mathrm{HCl} 2 \mathrm{M}$ and AcOEt, a solid was precipitated that was collected by filtration. The solid product was recrystallized by AcOEt to afford compound $5 \mathbf{b}$ (d.r. $>97: 3$ ) as a white solid in 54\% yield (90 mg, $0.22 \mathrm{mmol})$. m.p. $180-185^{\circ} \mathrm{C}$; $\mathrm{TLC} R_{\mathrm{f}}\left(\mathrm{CHCl}_{3} / \mathrm{MeOH} / \mathrm{AcOH}\right.$ : $7 / 2 / 1)=0.33 .{ }^{1} \mathrm{H}$ NMR $\left(200 \mathrm{MHz}, \mathrm{d}_{6}\right.$-DMSO + 4.5\%TFA) $\delta 7.52(\mathrm{~d}, J=9.0 \mathrm{~Hz}, 1 \mathrm{H})$, 7.39-7.13 (m, 10H), 5.11-4.96 (m, 2H), 3.85-3.72 (m, 1H), 3.00-2.79 (m, 3H), 2.04-1.94 (m, $1 \mathrm{H}), 1.78-1.61(\mathrm{~m}, 1 \mathrm{H}), 1.20(\mathrm{dd}, J=14.2,7.3 \mathrm{~Hz}, 3 \mathrm{H}) ;{ }^{13} \mathrm{C} \mathrm{NMR}\left(50 \mathrm{MHz}, \mathrm{d}_{6}-\mathrm{DMSO}+\right.$ $4.5 \%$ TFA) $\delta 175.4\left(\mathrm{~d},{ }^{3} J_{\mathrm{PC}}=9.4 \mathrm{~Hz}\right), 155.9\left(\mathrm{~d},{ }^{3} J_{\mathrm{PC}}=4.1 \mathrm{~Hz}\right), 138.9,137.1,129.1,128.5,128.3$, $127.9,127.8,126.4,65.7,46.2\left(\mathrm{~d},{ }^{1} J_{\mathrm{PC}}=105 \mathrm{~Hz}\right), 40.53,40.45,38.5,27.3\left(\mathrm{~d},{ }^{1} J_{\mathrm{PC}}=88 \mathrm{~Hz}\right)$, $13.9 ;{ }^{31} \mathrm{P}$ NMR $\left(81 \mathrm{MHz}, \mathrm{d}_{6}\right.$-DMSO + 4.5\%TFA) $\delta 47.6$; HRMS $(m / z):[\mathrm{M}+\mathrm{H}]^{+}$calcd. for $\mathrm{C}_{20} \mathrm{H}_{25} \mathrm{NO}_{6} \mathrm{P}^{+}, 406.1414$ found, 406.1424 .

3-\{[(R)-1-(Benzyloxycarbonylamino)-2-phenylethyl](hydroxy)phosphoryl\}-2-methylpropanoic acid (5c): Phosphinic acid $1 \mathrm{a}(250 \mathrm{mg}, 0.78 \mathrm{mmol})$ and methacrylic acid (3b) (81 $\mathrm{mg}, 0.94 \mathrm{mmol})$ were subjected to the general HMDS-mediated P-C bond-forming reaction protocol. After the addition of $\mathrm{HCl} 2 \mathrm{M}$ and $\mathrm{AcOEt}$, the two phases were separated and the organic layer was dried with $\mathrm{Na}_{2} \mathrm{SO}_{4}$ and evaporated in vacuo. The crude product was purified by silica gel column chromatography using $\mathrm{CHCl}_{3} / \mathrm{MeOH} / \mathrm{AcOH}$ 7:0.01:0:01 $\rightarrow$ 7:0.4:0:4 as eluent solvent system. Compound 5c (d.r. 2:1) was isolated as a white solid in $84 \%$ yield (266 mg, $0.65 \mathrm{mmol})$. TLC $R_{\mathrm{f}}\left(\mathrm{CHCl}_{3} / \mathrm{MeOH} / \mathrm{AcOH}: 7 / 2 / 1\right)=0.39 .{ }^{1} \mathrm{H} \mathrm{NMR}\left(200 \mathrm{MHz}, \mathrm{d}_{6}-\mathrm{DMSO}\right)$ $\delta 7.72 \& 7.71(2 \times \mathrm{d}, J=9.5 \mathrm{~Hz}, 1 \mathrm{H}), 7.36-7.07(\mathrm{~m}, 10 \mathrm{H}), 4.92(\mathrm{~s}, 2 \mathrm{H}), 3.97-3.73(\mathrm{~m}, 1 \mathrm{H})$, 3.18-2.94 (m, 1H), 2.84-2.57 (m, 2H), 2.19-1.93 (m, 1H), 1.73-1.45 (m, 1H), $1.19 \& 1.17(2 \times$ $\mathrm{d}, J=7.0 \mathrm{~Hz}, 3 \mathrm{H}) ;{ }^{13} \mathrm{C} \mathrm{NMR}\left(50 \mathrm{MHz}, \mathrm{d}_{6}\right.$-DMSO) $\delta 176.90,176.86,176.7,156.1,156.0,138.8$, $138.7,138.5,138.44,137.37,137.3,129.1,128.4,128.2,127.6,127.1,127.0,126.2,65.1,56.6(d$, $\left.{ }^{1} J_{\mathrm{PC}}=104 \mathrm{~Hz}\right), 52.2^{*}\left(\mathrm{~d},{ }^{1} J_{\mathrm{PC}}=105 \mathrm{~Hz}\right), 33.4,33.35,33.28,33.2,32.7,30.6,30.4,28.88,28.68$, $19.0\left(\mathrm{~d},{ }^{1} J_{\mathrm{PC}}=7.4 \mathrm{~Hz}\right), 18.7\left(\mathrm{~d},{ }^{1} J_{\mathrm{PC}}=6.0 \mathrm{~Hz}\right) ;{ }^{31} \mathrm{P} \mathrm{NMR}\left(81 \mathrm{MHz}, \mathrm{d}_{6}-\mathrm{DMSO}+5 \% \mathrm{TFA}\right) \delta$ 46.5; $\operatorname{HRMS}(\mathrm{m} / \mathrm{z})$ : $[\mathrm{M}-\mathrm{H}]^{-}$calcd. for $\mathrm{C}_{20} \mathrm{H}_{23} \mathrm{NO}_{6} \mathrm{P}^{-}, 404.1268$ found, 404.1264 .

(2S)-2-(\{[(R)-1-(Benzyloxycarbonylamino)-2-phenylethyl](hydroxy)phosphoryl\}methyl)-4-methylpe ntanoic acid (5d): Phosphinic acid 1a $(100 \mathrm{mg}, 0.31 \mathrm{mmol})$ and acrylic acid 3c (48 $\mathrm{mg}$, $0.37 \mathrm{mmol}$ ) were subjected to the general HMDS-mediated P-C bond-forming reaction protocol. After the addition of $\mathrm{HCl} 2 \mathrm{M}$ and AcOEt, the two phases are separated and the organic layer was dried with $\mathrm{Na}_{2} \mathrm{SO}_{4}$ and evaporated in vacuo. The residue was recrystallized twice with AcOEt to afford compound 5d (d.r. 95:5) as a white solid in $43 \%$ yield $(60 \mathrm{mg}, 0.13 \mathrm{mmol})$. m.p. $153-156^{\circ} \mathrm{C}$; $[\mathrm{a}]_{\mathrm{D}}{ }^{25}=-32(\mathrm{c}=0.31, \mathrm{DMSO})$; TLC $R_{\mathrm{f}}$ $\left(\mathrm{CHCl}_{3} / \mathrm{MeOH} / \mathrm{AcOH}: 7 / 2 / 1\right)=0.6 .{ }^{1} \mathrm{H} \mathrm{NMR}\left(200 \mathrm{MHz}, \mathrm{d}_{6}-\mathrm{DMSO}\right) \delta 7.66(\mathrm{~d}, J=9.5 \mathrm{~Hz}$, $1 \mathrm{H}), 7.39-6.88(\mathrm{~m}, 10 \mathrm{H}), 5.02-4.78(\mathrm{~m}, 2 \mathrm{H}), 4.02-3.73(\mathrm{~m}, 1 \mathrm{H}), 3.17-2.97(\mathrm{~m}, 1 \mathrm{H}), 2.82-2.57$ (m, 2H), 2.11-1.86 (m, 1H), 1.78-1.26 (m, 4H), 0.86 (app t, $J=6.3 \mathrm{~Hz}, 6 \mathrm{H}) ;{ }^{13} \mathrm{C} \mathrm{NMR}(50 \mathrm{MHz}$, $\mathrm{d}_{6}$-DMSO) $\delta 176.5\left(\mathrm{~d},{ }^{3} J_{\mathrm{PC}}=7.6 \mathrm{~Hz}\right), 156.1\left(\mathrm{~d},{ }^{3} J_{\mathrm{PC}}=3.8 \mathrm{~Hz}\right), 138.6,138.4,137.3,129.1$, $128.4,128.2,127.6,127.1,126.3,65.1,52.8\left(\mathrm{~d},{ }^{1} J_{\mathrm{PC}}=104 \mathrm{~Hz}\right), 42.7\left(\mathrm{~d}, J_{\mathrm{PC}}=9.0 \mathrm{~Hz}\right), 36.8(\mathrm{~d}$, $\left.J_{\mathrm{PC}}=4.0 \mathrm{~Hz}\right), 32.8,28.9\left(\mathrm{~d},{ }^{1} J_{\mathrm{PC}}=88 \mathrm{~Hz}\right), 25.60,23.01,21.87 ;{ }^{31} \mathrm{P} \mathrm{NMR}\left(81 \mathrm{MHz}, \mathrm{d}_{6}-\mathrm{DMSO}\right)$ $\delta 45.7^{*}, 45.4$; HRMS (m/z): $[\mathrm{M}-\mathrm{H}]^{-}$calcd. for $\mathrm{C}_{23} \mathrm{H}_{29} \mathrm{NO}_{6} \mathrm{P}^{-}, 446.1738$ found, 446.1739. 
(2S)-2-(\{[(R)-1-(Benzyloxycarbonylamino)-2-phenylethyl](hydroxy)phosphoryl\}methyl)-4-phe nylbutanoic acid (5e): Phosphinic acid $\mathbf{1 a}(200 \mathrm{mg}, 0.63 \mathrm{mmol})$ and acrylic acid $3 \mathbf{d}(133 \mathrm{mg}, 0.76$ $\mathrm{mmol}$ ) were subjected to the general HMDS-mediated P-C bond-forming reaction protocol. After the addition of $\mathrm{HCl} 2 \mathrm{M}$ and $\mathrm{AcOEt}$, a solid was precipitated that was collected by filtration. The solid product was recrystallized by AcOEt to afford compound 5e (d.r. 98:2) as a white solid in $47 \%$ yield $(147 \mathrm{mg}, 0.30 \mathrm{mmol})$. m.p. $196-199^{\circ} \mathrm{C}$; [a $]_{\mathrm{D}}{ }^{25}=-31.2(\mathrm{c}=0.9$, DMSO); TLC $R_{\mathrm{f}}\left(\mathrm{CHCl}_{3} / \mathrm{MeOH} / \mathrm{AcOH}: 7 / 2 / 1\right)=0.62 .{ }^{1} \mathrm{H}$ NMR $\left(200 \mathrm{MHz}, \mathrm{d}_{6}-\mathrm{DMSO}\right) \delta$ $7.71(\mathrm{~d}, J=9.7 \mathrm{~Hz}, 1 \mathrm{H}), 7.40-6.90(\mathrm{~m}, 15 \mathrm{H}), 4.92(\mathrm{~s}, 2 \mathrm{H}), 3.98-3.73(\mathrm{~m}, 1 \mathrm{H}), 3.20-2.95(\mathrm{~m}$, $1 \mathrm{H}), 2.82-2.41\left(\mathrm{~m}, 4 \mathrm{H}\right.$, overlapped by $\left.\mathrm{d}_{6}-\mathrm{DMSO}\right), 2.17-1.62(\mathrm{~m}, 4 \mathrm{H}) ;{ }^{13} \mathrm{C} \mathrm{NMR}(50 \mathrm{MHz}$, $\mathrm{d}_{6}$-DMSO) $\delta 175.9\left(\mathrm{~d},{ }^{3} J_{\mathrm{PC}}=9.0 \mathrm{~Hz}\right), 156.1\left(\mathrm{~d},{ }^{3} J_{\mathrm{PC}}=3.7 \mathrm{~Hz}\right), 141.5,138.6,138.4,137.3$, $129.1,128.4,128.3,128.2,127.6,127.1,126.3,125.9,65.1,52.7\left(\mathrm{~d},{ }^{1} J_{\mathrm{PC}}=104 \mathrm{~Hz}\right), 38.4(\mathrm{~d}$, $\left.J_{\mathrm{PC}}=4.2 \mathrm{~Hz}\right), 34.8\left(\mathrm{~d}, J_{\mathrm{PC}}=8.0 \mathrm{~Hz}\right), 32.8,32.5,28.1\left(\mathrm{~d},{ }^{1} J_{\mathrm{PC}}=89 \mathrm{~Hz}\right) ;{ }^{31} \mathrm{P}$ NMR $(81 \mathrm{MHz}$, $\mathrm{d}_{6}$-DMSO + 2\% TFA) $\delta 46.2$; HRMS $(m / z)$ : $[\mathrm{M}-\mathrm{H}]^{-}$calcd. for $\mathrm{C}_{24} \mathrm{H}_{29} \mathrm{NO}_{6} \mathrm{P}^{-}, 494.1738$ found, 494.1744 .

(2S)-2-(\{[(R)-1-(Benzyloxycarbonylamino)-2-phenylethyl](hydroxy)phosphoryl\}methyl)-5-phe nylpentanoic acid (5f): Phosphinic acid 1a (200 mg, $0.63 \mathrm{mmol})$ and acrylic acid $3 \mathbf{e}(143 \mathrm{mg}, 0.75 \mathrm{mmol})$ were subjected to the general HMDS-mediated P-C bond-forming reaction protocol. After the addition of $\mathrm{HCl} 2 \mathrm{M}$ and $\mathrm{AcOEt}$, the two phases were separated and the organic layer was dried with $\mathrm{Na}_{2} \mathrm{SO}_{4}$ and evaporated in vacuo. The residue was recrystallized twice with AcOEt to afford compound $\mathbf{5 f}$ (d.r. 98:2) as a white solid in $61 \%$ yield $(196 \mathrm{mg}, 0.38 \mathrm{mmol})$. m.p. $183-186^{\circ} \mathrm{C} ;[\mathrm{a}]_{\mathrm{D}}{ }^{25}=-22.0(\mathrm{c}=1, \mathrm{AcOH}) ; \mathrm{TLC} R_{\mathrm{f}}\left(\mathrm{CHCl}_{3} / \mathrm{MeOH} / \mathrm{AcOH}: 7 / 2 / 1\right)=0.60$. ${ }^{1} \mathrm{H} \mathrm{NMR}\left(200 \mathrm{MHz}, \mathrm{d}_{6}\right.$-DMSO) $\delta 7.71(\mathrm{~d}, J=9.0 \mathrm{~Hz}, 1 \mathrm{H}), 7.44-6.96(\mathrm{~m}, 15 \mathrm{H}), 4.98-4.72(\mathrm{~m}, 2 \mathrm{H})$, 3.95-3.72 (m, 1H), 3.14-2.96 (m, 1H), 2.80-2.33 (m, 4H, overlapped by d $\mathrm{d}_{6}$-DMSO), 2.10-1.81 $(\mathrm{m}, 1 \mathrm{H}), 1.79-1.35(\mathrm{~m}, 5 \mathrm{H}) ;{ }^{13} \mathrm{C}$ NMR $\left(50 \mathrm{MHz}, \mathrm{d}_{6}\right.$-DMSO) $\delta 176.0\left(\mathrm{~d},{ }^{3} J_{\mathrm{PC}}=7.3 \mathrm{~Hz}\right), 156.0(\mathrm{~d}$, $\left.{ }^{3} J_{\mathrm{PC}}=3.6 \mathrm{~Hz}\right), 142.0,138.7,138.4,137.3,129.0,128.3,128.2,127.5,126.9,126.2,125.7,65.0,52.8$ $\left(\mathrm{d},{ }^{1} J_{\mathrm{PC}}=104 \mathrm{~Hz}\right), 35.0,33.0,32.8,28.6,28.5\left(\mathrm{~d},{ }^{1} J_{\mathrm{PC}}=104 \mathrm{~Hz}\right) ;{ }^{31} \mathrm{P} \mathrm{NMR}\left(81 \mathrm{MHz}, \mathrm{d}_{6}-\mathrm{DMSO}\right)$ $\delta$ 45.0; HRMS $(\mathrm{m} / \mathrm{z})$ : $[\mathrm{M}-\mathrm{H}]^{-}$calcd. for $\mathrm{C}_{28} \mathrm{H}_{31} \mathrm{NO}_{6} \mathrm{P}^{-}, 508.1894$ found, 508.1889.

(2S)-2-(\{[(R)-1-(Benzyloxycarbonylamino)-2-phenylethyl](hydroxy)phosphoryl\}methyl)pent-4-enoic acid (5g): Phosphinic acid 1a (200 mg, $0.63 \mathrm{mmol})$ and acrylic acid $3 \mathrm{f}(84 \mathrm{mg}, 0.75 \mathrm{mmol})$ were subjected to the general HMDS-mediated P-C bond-forming reaction protocol. After the addition of $\mathrm{HCl} 2 \mathrm{M}$ and $\mathrm{AcOEt}$, the two phases were separated and the organic layer was dried with $\mathrm{Na}_{2} \mathrm{SO}_{4}$ and evaporated in vacuo. The crude product was purified by silica gel column chromatography using $\mathrm{CHCl}_{3} / \mathrm{MeOH} / \mathrm{AcOH}$ 7:0.01:0:01 $\rightarrow$ 7:0.4:0:4 as eluent solvent system. Compound $\mathbf{5 g}$ was isolated as an off-white solid in $94 \%$ yield $(255 \mathrm{mg}, 0.59 \mathrm{mmol})$. Isolation of diastereoisomer $(R, S)-5 \mathrm{~g}$ (d.r. 99:1) was achieved after 3 recrystallizations with $\mathrm{CHCl}_{3}$ in $23 \%$ yield $(62 \mathrm{mg}, 0.14 \mathrm{mmol})$. m.p. $131-133{ }^{\circ} \mathrm{C}$; $[\mathrm{a}]_{\mathrm{D}}{ }^{25}=-32.8(\mathrm{c}=1, \mathrm{MeOH}) ; \mathrm{TLC} R_{\mathrm{f}}\left(\mathrm{CHCl}_{3} / \mathrm{MeOH} / \mathrm{AcOH}: 7 / 2 / 1\right)=0.44 .{ }^{1} \mathrm{H}$ NMR $(200$ $\mathrm{MHz}, \mathrm{d}_{6}$-DMSO) $\delta 7.70(\mathrm{~d}, J=9.2 \mathrm{~Hz}, 1 \mathrm{H}), 7.39-7.03(\mathrm{~m}, 10 \mathrm{H}), 5.81-5.55(\mathrm{~m}, 1 \mathrm{H}), 5.11-4.96$ $(\mathrm{m}, 2 \mathrm{H}), 4.92(\mathrm{~s}, 2 \mathrm{H}), 3.98-3.74(\mathrm{~m}, 1 \mathrm{H}), 3.15-2.94(\mathrm{~m}, 1 \mathrm{H}), 2.86-2.59(\mathrm{~m}, 2 \mathrm{H}), 2.42-2.23$ $(\mathrm{m}, 2 \mathrm{H}), 2.10-1.87(\mathrm{~m}, 1 \mathrm{H}), 1.83-1.59(\mathrm{~m}, 1 \mathrm{H}) ;{ }^{13} \mathrm{C}$ NMR $\left(50 \mathrm{MHz}, \mathrm{d}_{6}\right.$-DMSO) $\delta 175.4(\mathrm{~d}$, $\left.{ }^{3} J_{\mathrm{PC}}=8.9 \mathrm{~Hz}\right), 156.1\left(\mathrm{~d},{ }^{3} J_{\mathrm{PC}}=3.8 \mathrm{~Hz}\right), 138.6,138.4,137.3,135.1,129.0,128.3,128.2,127.6$, $127.1,126.2,117.5,52.6\left(\mathrm{~d},{ }^{1} J_{\mathrm{PC}}=105 \mathrm{~Hz}\right), 36.8\left(\mathrm{~d}, J_{\mathrm{PC}}=7.7 \mathrm{~Hz}\right), 32.7,27.3\left(\mathrm{~d},{ }^{1} J_{\mathrm{PC}}=89 \mathrm{~Hz}\right)$; ${ }^{31} \mathrm{P}$ NMR (81 MHz, $\mathrm{d}_{6}$-DMSO) $\delta 45.7$; HRMS $(\mathrm{m} / \mathrm{z})$ : $[\mathrm{M}-\mathrm{H}]^{-}$calcd. for $\mathrm{C}_{23} \mathrm{H}_{25} \mathrm{NO}_{6} \mathrm{P}^{-}$, 430.1425 found, 430.1437 .

(2S)-2-(\{[(R)-1-(Benzyloxycarbonylamino)-2-phenylethyl](hydroxy)phosphoryl\}methyl)pent-4-ynoic acid (5h): Phosphinic acid 1a (410 g, $1.3 \mathrm{mmol})$ and acrylic acid $3 \mathrm{~g}$ (170 mg, $1.54 \mathrm{mmol})$ were subjected to the general HMDS-mediated P-C bond-forming reaction protocol. After the addition of $\mathrm{HCl} 2 \mathrm{M}$ and $\mathrm{AcOEt}$, a solid was precipitated that was collected by filtration. The solid product was refluxed with AcOEt and the hot suspension is filtrated. The procedure is repeated to afford compound $5 \mathrm{~h}$ (d.r. $96: 4)$ as a white solid in $51 \%$ yield $(284 \mathrm{mg}$, $0.66 \mathrm{mmol})$. m.p. $171-174^{\circ} \mathrm{C}$; $[\mathrm{a}]_{\mathrm{D}}{ }^{25}=-43\left(\mathrm{c}=1\right.$, DMSO); $\mathrm{TLC} R_{\mathrm{f}}\left(\mathrm{CHCl}_{3} / \mathrm{MeOH} / \mathrm{AcOH}\right.$ : $7 / 2 / 1)=0.59 .{ }^{1} \mathrm{H}$ NMR $\left(400 \mathrm{MHz}, \mathrm{d}_{6}\right.$-DMSO) $\delta 7.70(\mathrm{~d}, J=9.4 \mathrm{~Hz}, 1 \mathrm{H}), 7.36-6.94(\mathrm{~m}, 10 \mathrm{H})$, $4.95(\mathrm{~d}, J=13 \mathrm{~Hz}, 1 \mathrm{H}), 4.90(\mathrm{~d}, J=13 \mathrm{~Hz}, 1 \mathrm{H}), 4.01-3.84(\mathrm{~m}, 1 \mathrm{H}), 3.17-3.02(\mathrm{~m}, 2 \mathrm{H}), 2.92-2.51$ 
(m, 5H), 2.20-2.04 (m, 1H), 1.96-1.81 (m, 1H); ${ }^{13} \mathrm{C} \mathrm{NMR} \mathrm{(101} \mathrm{MHz,} \mathrm{d}_{6}$-DMSO) $\delta 174.3(\mathrm{~d}$, $\left.{ }^{3} J_{\mathrm{PC}}=11.7 \mathrm{~Hz}\right), 156.1\left(\mathrm{~d},{ }^{3} \mathrm{JPC}_{\mathrm{PC}}=3.5 \mathrm{~Hz}\right), 138.5,138.3,137.2,129.0,128.3,128.2,127.6,127.1$, 126.7, 126.2, 81.4, 72.9, 65.2, $52.3\left(\mathrm{~d},{ }^{1} J_{\mathrm{PC}}=105 \mathrm{~Hz}\right), 37.8,32.7,26.7\left(\mathrm{~d},{ }^{1} J_{\mathrm{PC}}=89 \mathrm{~Hz}\right), 21.5(\mathrm{~d}$, $\left.{ }^{1} J_{\mathrm{PC}}=4.9 \mathrm{~Hz}\right) ;{ }^{31} \mathrm{P}$ NMR $\left(162 \mathrm{MHz}, \mathrm{d}_{6}\right.$-DMSO) $\delta 45.0 ; \mathrm{HRMS}(\mathrm{m} / z):[\mathrm{M}-\mathrm{H}]^{-}$calcd. for $\mathrm{C}_{23} \mathrm{H}_{23} \mathrm{NO}_{6} \mathrm{P}^{-}$, 428.1268 found, 428.1262 .

(2S)-3-\{[1,1'-Biphenyl]-4-yl\}-2-(\{[(R)-1-(benzyloxycarbonylamino)-2-phenylethyl](hydroxy)phospho ryl\}methyl)propanoic acid (5i): Phosphinic acid $\mathbf{1 a}(200 \mathrm{mg}, 0.63 \mathrm{mmol})$ and acrylic acid $3 \mathbf{h}$ $(180 \mathrm{mg}, 0.76 \mathrm{mmol})$ were subjected to the general HMDS-mediated P-C bond-forming reaction protocol. After the addition of $\mathrm{HCl} 2 \mathrm{M}$ and $\mathrm{AcOEt}$, a solid was precipitated that was collected by filtration. The solid product was refluxed with AcOEt and the hot suspension is filtrated. The procedure was repeated to afford compound $5 \mathbf{i}$ (d.r. $>99: 1$ ) as a white solid in $68 \%$ yield $(239 \mathrm{~g}, 0.43 \mathrm{mmol})$. m.p. $208-210{ }^{\circ} \mathrm{C}$; $[\mathrm{a}]_{\mathrm{D}}{ }^{25}=-23.8(\mathrm{c}=0.95, \mathrm{DMSO}) ;{ }^{1} \mathrm{H}$ NMR $\left(200 \mathrm{MHz}, \mathrm{d}_{6}\right.$-DMSO + 4\%TFA) $\delta 7.77-6.95(\mathrm{~m}, 2 \mathrm{H}), 5.02-4.74(\mathrm{~m}, 2 \mathrm{H}), 4.03-3.77(\mathrm{~m}$, $1 \mathrm{H}), 3.16-2.82(\mathrm{~m}, 4 \mathrm{H}), 2.81-2.59(\mathrm{~m}, 1 \mathrm{H}), 2.19-1.93(\mathrm{~m}, 1 \mathrm{H}), 1.92-1.67(\mathrm{~m}, 1 \mathrm{H}) ;{ }^{13} \mathrm{C} \mathrm{NMR}$ (50 MHz, $\mathrm{d}_{6}$-DMSO) $\delta 175.3\left(\mathrm{~d},{ }^{3} J_{\mathrm{PC}}=9.6 \mathrm{~Hz}\right), 156.0\left(\mathrm{~d},{ }^{3} J_{\mathrm{PC}}=3.8 \mathrm{~Hz}\right), 140.0,138.6,138.3$, 138.2, 137.2, 129.7, 129.0, 129.0, 128.3, 128.2, 127.6, 127.3, 127.0, 126.6, 126.2, 65.1, 52.6, (d, $\left.{ }^{1} J_{\mathrm{PC}}=105 \mathrm{~Hz}\right), 32.71,27.7\left(\mathrm{~d},{ }^{1} J_{\mathrm{PC}}=89 \mathrm{~Hz}\right) ;{ }^{31} \mathrm{P} \mathrm{NMR}\left(81 \mathrm{MHz}, \mathrm{d}_{6}-\mathrm{DMSO}+4 \% \mathrm{TFA}\right) \delta 46.6$; $\operatorname{HRMS}(\mathrm{m} / \mathrm{z}):[\mathrm{M}+\mathrm{Na}]^{+}$calcd. for $\mathrm{C}_{32} \mathrm{H}_{31} \mathrm{NO}_{6} \mathrm{P}^{-}, 556.1894$ found, 556.1896.

(2S)-2-(\{[(R)-1-(Benzyloxycarbonylamino)-2-phenylethyl](hydroxy)phosphoryl\}methyl)-4-(1,3-dioxolan2-yl)butanoic acid (5j): Phosphinic acid 1a (200 mg, $0.63 \mathrm{mmol})$ and acrylic acid $3 \mathbf{i}$ (130 mg, $0.76 \mathrm{mmol}$ ) were subjected to the general HMDS-mediated P-C bond-forming reaction protocol. After the addition of $\mathrm{HCl} 2 \mathrm{M}$ and AcOEt, a solid was precipitated that was collected by filtration. The solid product was recrystallized by AcOEt to afford compound 5j (d.r. 99:1) as a white solid in $54 \%$ yield $(167 \mathrm{mg}, 0.34 \mathrm{mmol})$. m.p. $143-146^{\circ} \mathrm{C}$; $[\mathrm{a}]_{\mathrm{D}}{ }^{25}=-31.0(\mathrm{c}=0.9$, $\mathrm{MeOH})$; TLC $R_{\mathrm{f}}\left(\mathrm{CHCl}_{3} / \mathrm{MeOH} / \mathrm{AcOH}: 7 / 2 / 1\right)=0.34 .{ }^{1} \mathrm{H}$ NMR $\left(200 \mathrm{MHz}, \mathrm{d}_{6}-\mathrm{DMSO}\right) \delta$ $7.69(\mathrm{~d}, J=9.4 \mathrm{~Hz}, 1 \mathrm{H}), 7.39-6.92(\mathrm{~m}, 10 \mathrm{H}), 4.92(\mathrm{~s}, 2 \mathrm{H}), 4.79-4.70(\mathrm{~m}, 1 \mathrm{H}), 3.96-3.67(\mathrm{~m}, 5 \mathrm{H})$, 3.14-2.97 (m, 1H), 2.81-2.56 (m, 2H), 2.12-1.88 (m, 1H), $1.81-1.43(\mathrm{~m}, 5 \mathrm{H}) ;{ }^{13} \mathrm{C}$ NMR $(50 \mathrm{MHz}$, $\mathrm{d}_{6}$-DMSO) $\delta 175.9\left(\mathrm{~d},{ }^{3} J_{\mathrm{PC}}=8.3 \mathrm{~Hz}\right), 156.1\left(\mathrm{~d},{ }^{3} J_{\mathrm{PC}}=3.7 \mathrm{~Hz}\right), 138.6,138.4,137.3,129.0,128.3$, 128.2, 127.6, 127.1, 126.3, 103.3, 65.1, 64.3, $52.7\left(\mathrm{~d},{ }^{1} J_{\mathrm{PC}}=105 \mathrm{~Hz}\right), 38.4\left(\mathrm{~d}, J_{\mathrm{PC}}=3.3 \mathrm{~Hz}\right), 32.8$, $30.9,28.2\left(\mathrm{~d},{ }^{1} J_{\mathrm{PC}}=88 \mathrm{~Hz}\right), 27.5\left(\mathrm{~d}, J_{\mathrm{PC}}=7.5 \mathrm{~Hz}\right) ;{ }^{1}{ }^{\mathrm{P}} \mathrm{NMR}\left(81 \mathrm{MHz}, \mathrm{d}_{6}\right.$-DMSO) $\delta 45.6 ; \mathrm{HRMS}$ $(\mathrm{m} / \mathrm{z}):[\mathrm{M}-\mathrm{H}]^{-}$calcd. for $\mathrm{C}_{24} \mathrm{H}_{29} \mathrm{NO}_{6} \mathrm{P}^{-}, 490.1636$ found, 490.1638 .

(2S)-2-(\{[(R)-1-(Benzyloxycarbonylamino)-2-phenylethyl](hydroxy)phosphoryl\}methyl)-5-[(tert-but oxycarbonyl)amino]pentanoic acid (5k): Phosphinic acid $\mathbf{1 a}(300 \mathrm{mg}, 0.94 \mathrm{mmol})$ and acrylic acid 3j ( $259 \mathrm{mg}, 1.12 \mathrm{mmol}$ ) were subjected to the general HMDS-mediated P-C bond-forming reaction protocol. After the addition of $\mathrm{HCl} 2 \mathrm{M}$ and $\mathrm{AcOEt}$, the two phases were separated and the organic layer was dried with $\mathrm{Na}_{2} \mathrm{SO}_{4}$ and evaporated in vacuo. The crude product was purified by silica gel column chromatography using $\mathrm{CHCl}_{3} / \mathrm{MeOH} / \mathrm{AcOH}$ 7:0.01:0:01 $\rightarrow$ 7:0.4:0:4 as eluent solvent system. Compound 5k (d.r. 3.5:1) was isolated as a white foam in $87 \%$ yield (449 mg, $0.82 \mathrm{mmol})$. TLC $\mathrm{R}_{\mathrm{f}}\left(\mathrm{CHCl}_{3} / \mathrm{MeOH} / \mathrm{AcOH}: 7 / 2 / 1\right)=0.72 .{ }^{1} \mathrm{H} \mathrm{NMR}(200 \mathrm{MHz}$, $\mathrm{d}_{6}$-DMSO) $\delta 7.63(\mathrm{~d}, \mathrm{~J}=9.5 \mathrm{~Hz}, 1 \mathrm{H}), 7.35-6.70(\mathrm{~m}, 10 \mathrm{H}), 4.93(\mathrm{~d}, \mathrm{~J}=13 \mathrm{~Hz}, 1 \mathrm{H}), 4.84(\mathrm{~d}, \mathrm{~J}=13 \mathrm{~Hz}$, $1 \mathrm{H}), 3.93-3.69(\mathrm{~m}, 1 \mathrm{H}), 3.12-2.93(\mathrm{~m}, 1 \mathrm{H}), 2.93-2.52$ (m, 5H, overlapped by d 6 -DMSO), 2.15-1.82 $(\mathrm{m}, 1 \mathrm{H}), 1.74-1.14(\mathrm{~m}, 13 \mathrm{H}) ;{ }^{13} \mathrm{C}$ NMR $\left(50 \mathrm{MHz}, \mathrm{d}_{6}-\mathrm{DMSO} / \mathrm{CDCl}_{3} 1: 1\right) \delta 176.0\left(\mathrm{~d},{ }^{3} \mathrm{~J}_{\mathrm{PC}}=8.1\right.$ $\mathrm{Hz}), 156.0\left(\mathrm{~d},{ }^{3} \mathrm{~J}_{\mathrm{PC}}=3.6 \mathrm{~Hz}\right), 155.6,138.6,138.3,137.2,129.0,128.3,128.1,127.5,127.0,126.1,65.1$, $52.6\left(\mathrm{~d},{ }^{1} \mathrm{JPC}_{\mathrm{PC}}=105 \mathrm{~Hz}\right), 32.8,30.6,30.5,29.1,28.8^{*}, 28.7^{*}, 28.3,28.0^{*}, 27.2 ;{ }^{31} \mathrm{P}$ NMR $(81 \mathrm{MHz}$, $\mathrm{d}_{6}$-DMSO) $\delta 46.3^{*}, 45.9$; HRMS (m/z): $[\mathrm{M}-\mathrm{H}]^{-}$calcd. for $\mathrm{C}_{27} \mathrm{H}_{36} \mathrm{~N}_{2} \mathrm{O}_{8} \mathrm{P}^{-}, 547.2215$ found, 547.2217.

2-(\{[(R)-1-(Benzyloxycarbonylamino)ethyl](hydroxy)phosphoryl $\}$ methyl)-4-(tert-butoxy)-4-oxo-2 $\lambda^{3}$. butanoic acid (51): Phosphinic acid $\mathbf{1 b}(500 \mathrm{mg}, 1.57 \mathrm{mmol})$ and acrylic acid 3k (48 mg, $0.37 \mathrm{mmol}$ ) were subjected to the general HMDS-mediated P-C bond-forming reaction protocol. After the addition of $\mathrm{HCl} 2 \mathrm{M}$ and $\mathrm{AcOEt}$, the two phases were separated and the organic layer was dried with $\mathrm{Na}_{2} \mathrm{SO}_{4}$ and evaporated in vacuo. The residue was treated with $\mathrm{Et}_{2} \mathrm{O}$, filtrated and washed with $\mathrm{Et}_{2} \mathrm{O}$. Compound 51 (d.r. 2.5:1) was isolated as a white 
solid in 93\% yield (129 mg, $0.29 \mathrm{mmol})$. TLC $R_{\mathrm{f}}\left(\mathrm{CHCl}_{3} / \mathrm{MeOH} / \mathrm{AcOH}: 7 / 2 / 1\right)=0.28 .{ }^{1} \mathrm{H}$ $\operatorname{NMR}\left(200 \mathrm{MHz}, \mathrm{d}_{6}\right.$-DMSO) $\delta\left\{\left[7.58(\mathrm{~d}, J=9.0 \mathrm{~Hz}) \& 7.56^{*}(\mathrm{~d}, J=9.0 \mathrm{~Hz})\right], 1 \mathrm{H}\right\}, 7.41-7.25$ (m, 5H), 5.11-4.95 (m, 2H), 3.87-3.62 (m, 1H), 3.04-2.81 (m, 1H), 2.80-2.63 (m, 1H), 2.58-2.38 (m, 1H, overlapped by $\mathrm{d}_{6}$-DMSO), 2.13-1.92 (m, 1H), 1.83-1.60 (m, 1H), $1.37(\mathrm{~s}, 9 \mathrm{H}), 1.20$ $(\mathrm{dd}, J=14.4,7.4 \mathrm{~Hz}, 3 \mathrm{H}) ;{ }^{13} \mathrm{C} \mathrm{NMR}\left(50 \mathrm{MHz}, \mathrm{d}_{6}-\mathrm{DMSO}\right) \delta 175.3\left(\mathrm{~d},{ }^{3} J_{\mathrm{PC}}=13.5 \mathrm{~Hz}\right), 170.7$, $156.0\left(\mathrm{~d},{ }^{3} J_{\mathrm{PC}}=4.9 \mathrm{~Hz}\right), 128.5,128.0,127.84,127.79,80.0,65.8,46.3\left(\mathrm{~d},{ }^{1} J_{\mathrm{PC}}=105 \mathrm{~Hz}\right)^{*}, 46.1(\mathrm{~d}$, $\left.{ }^{1} J_{\mathrm{PC}}=107 \mathrm{~Hz}\right), 37.18^{*}, 37.16^{*}, 36.93,36.87,35.0^{*}, 34.9^{*}, 35.2,35.1,27.8,26.0,13.9 ;{ }^{31} \mathrm{P} \mathrm{NMR}$ (81 MHz, $\mathrm{d}_{6}$-DMSO) $\delta 47.3,46.9$; HRMS $(\mathrm{m} / \mathrm{z})$ : $[\mathrm{M}-\mathrm{H}]^{-}$calcd. for $\mathrm{C}_{19} \mathrm{H}_{27} \mathrm{NO}_{8} \mathrm{P}^{-}, 428.1480$ found, 428.1467 .

(2S)-2-((((R)-1-(((Benzyloxy)carbonyl)amino)-3-phenylpropyl)(hydroxy)phosphoryl)methyl)-4-meth ylpentanoic acid (5m): Phosphinic acid 1c $(1.0 \mathrm{~g}, 3.0 \mathrm{mmol})$ and acrylic acid 3c (461 mg, $3.6 \mathrm{mmol}$ ) were subjected to the general HMDS-mediated P-C bond-forming reaction protocol. After the addition of $\mathrm{HCl} 2 \mathrm{M}$ and $\mathrm{AcOEt}$, a solid was precipitated that was collected by filtration. The solid product was refluxed with AcOEt and the hot suspension was filtrated to afford compound $5 \mathrm{~m}$ (d.r. 96:4) as a white solid in 53\% yield (733 mg, $1.59 \mathrm{mmol}$ ). m.p. $184-186{ }^{\circ} \mathrm{C} ;[\mathrm{a}]_{\mathrm{D}}{ }^{25}=-19$ (c = 1, DMSO); TLC $R_{\mathrm{f}}\left(\mathrm{CHCl}_{3} / \mathrm{MeOH} / \mathrm{AcOH}: 7 / 2 / 1\right)=0.7 .{ }^{1} \mathrm{H}$ $\mathrm{NMR}\left(400 \mathrm{MHz}, \mathrm{d}_{6}-\mathrm{DMSO}+20 \% \mathrm{CD}_{3} \mathrm{OD}\right) \delta 7.57(\mathrm{~d}, J=9.2 \mathrm{~Hz}, 0.2 \mathrm{H}$ partially exchanged $)$, 7.41-7.08 (m, 10H), $5.08(\mathrm{~s}, 2 \mathrm{H}), 3.68-3.54(\mathrm{~m}, 1 \mathrm{H}), 2.77-2.57(\mathrm{~m}, 2 \mathrm{H}), 2.55-2.42(\mathrm{~m}, 1 \mathrm{H}$, overlapped by $\mathrm{d}_{6}$-DMSO), 2.03-1.86 (m, 2H), 1.85-1.72 (m, 1H), 1.67-1.56 (m, 1H), 1.55-1.28 $(\mathrm{m}, 3 \mathrm{H}), 0.85 \& 0.82(2 \times \mathrm{d}, J=6.4 \mathrm{~Hz}, 6 \mathrm{H}) ;{ }^{13} \mathrm{C} \mathrm{NMR}\left(101 \mathrm{MHz}, \mathrm{d}_{6}-\mathrm{DMSO}+20 \% \mathrm{CD}_{3} \mathrm{OD}\right)$ $\delta 176.4\left(\mathrm{~d},{ }^{3} J_{\mathrm{PC}}=7.8 \mathrm{~Hz}\right), 156.4\left(\mathrm{~d},{ }^{3} J_{\mathrm{PC}}=3.8 \mathrm{~Hz}\right), 141.3,137.3,128.5,128.4,128.3,127.9$, $127.7,125.9,65.6,50.3\left(\mathrm{~d},{ }^{1} J_{\mathrm{PC}}=105 \mathrm{~Hz}\right), 42.6\left(\mathrm{~d}, J_{\mathrm{PC}}=8.5 \mathrm{~Hz}\right), 36.7\left(\mathrm{~d}, J_{\mathrm{PC}}=3.9 \mathrm{~Hz}\right), 31.7$ $\left(\mathrm{d}, J_{\mathrm{PC}}=12 \mathrm{~Hz}\right), 29.3,28.8\left(\mathrm{~d},{ }^{1} J_{\mathrm{PC}}=89 \mathrm{~Hz}\right), 25.6,22.9,21.8 ;{ }^{31} \mathrm{P} \mathrm{NMR}\left(162 \mathrm{MHz}, \mathrm{d}_{6}-\mathrm{DMSO}\right.$ $\left.+20 \% \mathrm{CD}_{3} \mathrm{OD}\right) \delta 45.0$; HRMS $(\mathrm{m} / \mathrm{z}):[\mathrm{M}+\mathrm{H}]^{+}$calcd. for $\mathrm{C}_{24} \mathrm{H}_{33} \mathrm{NO}_{6} \mathrm{P}^{+}, 462.2040$ found, 462.2046 .

(2S)-2-(\{[(R)-1-(Benzyloxycarbonylamino)-3-phenylpropyl](hydroxy)phosphoryl\}methyl)pent-4-ynoic acid (5n): Phosphinic acid 1c (1.5 g, $4.5 \mathrm{mmol})$ and acrylic acid $3 \mathrm{~g}$ (594 mg, $5.4 \mathrm{mmol})$ were subjected to the general HMDS-mediated P-C bond-forming reaction protocol. After the addition of $\mathrm{HCl} 2 \mathrm{M}$ and $\mathrm{AcOEt}$, a solid was precipitated that was collected by filtration. The solid product was refluxed with AcOEt and the hot suspension was filtrated. The procedure was repeated thrice to afford compound 5 n (d.r. 95:5) as a white solid in 56\% yield (1.1 g, $2.5 \mathrm{mmol})$. m.p. $162-164{ }^{\circ} \mathrm{C}$; $[\mathrm{a}]_{\mathrm{D}}{ }^{25}=-22$ (c = 1, DMSO); TLC $R_{\mathrm{f}}\left(\mathrm{CHCl}_{3} / \mathrm{MeOH} / \mathrm{AcOH}\right.$ : $7 / 0.5 / 0.5)=0.16 .{ }^{1} \mathrm{H}$ NMR $\left(400 \mathrm{MHz}, \mathrm{d}_{6}-\mathrm{DMSO}+5 \%\right.$ TFA) $\delta 7.63(\mathrm{~d}, J=9.3 \mathrm{~Hz}, 1 \mathrm{H}), 7.42-7.08$ $(\mathrm{m}, 10 \mathrm{H}), 5.06(\mathrm{~s}, 2 \mathrm{H}), 3.73-3.57(\mathrm{~m}, 1 \mathrm{H}), 2.84-2.61(\mathrm{~m}, 2 \mathrm{H}), 2.62-2.43(\mathrm{~m}, 2 \mathrm{H}$, overlapped by $\mathrm{d}_{6}$-DMSO), 2.10-1.92 (m, 2H), 1.87-1.72 (m, 2H); ${ }^{13} \mathrm{C} \mathrm{NMR} \mathrm{(50} \mathrm{MHz,} \mathrm{d}_{6}$-DMSO) $\delta 174.6(\mathrm{~d}$, $\left.{ }^{3} J_{\mathrm{PC}}=11.8 \mathrm{~Hz}\right), 156.7\left(\mathrm{~d},{ }^{3} \mathrm{~J}_{\mathrm{PC}}=3.7 \mathrm{~Hz}\right), 141.5,137.4,128.7,128.7,128.2,128.0,126.2,81.6$, $73.1,66.0,50.3\left(\mathrm{~d},{ }^{1} J_{\mathrm{PC}}=106 \mathrm{~Hz}\right), 37.9,31.9\left(\mathrm{~d}, J_{\mathrm{PC}}=12 \mathrm{~Hz}\right), 29.4,26.7\left(\mathrm{~d},{ }^{1} J_{\mathrm{PC}}=87 \mathrm{~Hz}\right)$, 21.8; ${ }^{31} \mathrm{P}$ NMR (162 MHz, $\mathrm{d}_{6}$-DMSO + 5\% TFA) $\delta 45.7 ; \mathrm{HRMS}(\mathrm{m} / \mathrm{z}):[\mathrm{M}+\mathrm{Na}]^{+}$calcd. for $\mathrm{C}_{23} \mathrm{H}_{26} \mathrm{NNaO}_{6} \mathrm{P}^{+}, 466.1390$ found, 456.1368 .

2-[(\{(R)-1-[(tert-Butoxycarbonyl)amino]-3-phenylpropyl\}(hydroxy)phosphoryl)methyl]pent-4-ynoic acid (5o): Phosphinic acid 1d (450 mg, $1.50 \mathrm{mmol})$ and acrylic acid 3g (198 mg, $1.80 \mathrm{mmol})$ were subjected to the general HMDS-mediated P-C bond-forming reaction protocol. After the addition of $\mathrm{HCl} 2 \mathrm{M}$ and $\mathrm{AcOEt}$, the two phases were separated and the organic layer was dried with $\mathrm{Na}_{2} \mathrm{SO}_{4}$ and evaporated in vacuo. The crude product was purified by silica gel column chromatography using $\mathrm{CHCl}_{3} / \mathrm{MeOH} / \mathrm{AcOH}$ 7:0.01:0:01 $\rightarrow$ 7:0.4:0:4 as eluent solvent system. Compound 5o (d.r. 1.3:1) was isolated as an off-white solid in $89 \%$ yield $(547 \mathrm{mg}, 1.34 \mathrm{mmol})$. TLC $R_{\mathrm{f}}\left(\mathrm{CHCl}_{3} / \mathrm{MeOH} / \mathrm{AcOH}: 7 / 0.5 / 0.5\right)=0.16 .{ }^{1} \mathrm{H} \mathrm{NMR}$ (400 MHz, d $\mathrm{d}_{6}$-DMSO) $\delta$ 7.35-7.07 (m, 6H), 3.62-3.52 (m, 1H), 2.80-2.65 (m, 3H), 2.61-2.50 (m, 2H, overlapped by d 6 -DMSO), 2.06-1.89 (m, 2H), 1.84-1.68 (m, 2H), $1.41 \& 1.37(2 \times \mathrm{s}$, $9 \mathrm{H}) ;{ }^{13} \mathrm{C}$ NMR (101 MHz, d 6 -DMSO) $\delta 174.2\left(\mathrm{~d},{ }^{3} J_{\mathrm{PC}}=12 \mathrm{~Hz}\right), 155.7,155.64,155.61,141.39$, $141.36,128.5,128.3,125.8,81.4,78.3^{*}, 78.2,72.7,48.2\left(\mathrm{~d},{ }^{1} J_{\mathrm{PC}}=107 \mathrm{~Hz}\right), 37.8^{*}\left(\mathrm{~d}, J_{\mathrm{PC}}=2.7\right.$ $\mathrm{Hz}), 37.6\left(\mathrm{~d}, J_{\mathrm{PC}}=2.6 \mathrm{~Hz}\right), 31.7\left(\mathrm{~d}, J_{\mathrm{PC}}=12 \mathrm{~Hz}\right), 31.74,31.62,29.0,28.2,27.9,26.4^{*}\left(\mathrm{~d},{ }^{1} J_{\mathrm{PC}}=\right.$ $89 \mathrm{~Hz}), 26.5\left(\mathrm{~d},{ }^{1} J_{\mathrm{PC}}=89 \mathrm{~Hz}\right), 21.8^{*}\left(\mathrm{~d}, J_{\mathrm{PC}}=6.3 \mathrm{~Hz}\right), 21.4\left(\mathrm{~d}, J_{\mathrm{PC}}=5.7 \mathrm{~Hz}\right) ;{ }^{31} \mathrm{P} \mathrm{NMR}(162$ 
$\mathrm{MHz}, \mathrm{d}_{6}$-DMSO) $\delta$ 45.7, 45.4; HRMS ( $\left.m / z\right):[\mathrm{M}+\mathrm{Na}]^{+}$calcd. for $\mathrm{C}_{20} \mathrm{H}_{28} \mathrm{NNaO}_{6} \mathrm{P}^{+}, 432.1546$ found, 432.1555 .

(2S)-2-(\{[(R)-1-(Benzyloxycarbonylamino)-3-(phenylthio)propyl](hydroxy)phosphoryl\}methyl)-4-me thylpentanoic acid (5p): Phosphinic acid 1e $(2.0 \mathrm{~g}, 5.5 \mathrm{mmol})$ and acrylic acid $3 \mathrm{c}(840 \mathrm{mg}$, $6.6 \mathrm{mmol}$ ) were subjected to the general HMDS-mediated P-C bond-forming reaction protocol. After the addition of $\mathrm{HCl} 2 \mathrm{M}$ and $\mathrm{AcOEt}$, a solid was precipitated that was collected by filtration. The solid product was refluxed with AcOEt and the hot suspension was filtrated. The procedure was repeated to afford compound $5 p$ (d.r. 97:3) as a white solid in $59 \%$ yield $(1.6 \mathrm{~g}, 2.5 \mathrm{mmol})$. m.p. $130-135^{\circ} \mathrm{C}$; $[\mathrm{a}]_{\mathrm{D}}{ }^{25}=-14.2(\mathrm{c}=1, \mathrm{DMSO})$; TLC $R_{\mathrm{f}}$ $\left(\mathrm{CHCl}_{3} / \mathrm{MeOH} / \mathrm{AcOH}: 7 / 2 / 1\right)=0.65 .{ }^{1} \mathrm{H}$ NMR $\left(500 \mathrm{MHz}, \mathrm{d}_{6}\right.$-DMSO + 3\%TFA) $\delta 7.65$ $(\mathrm{d}, J=9.5 \mathrm{~Hz}, 1 \mathrm{H}), 7.42-7.08(\mathrm{~m}, 10 \mathrm{H}), 5.06(\mathrm{~s}, 2 \mathrm{H}), 3.97-3.74(\mathrm{~m}, 1 \mathrm{H}), 3.16-2.78(\mathrm{~m}, 2 \mathrm{H})$, 2.74-2.54 (m, 1H, overlapped by $\mathrm{d}_{6}$-DMSO), 2.01-1.74 (m,3H), 1.72-1.23 (m, 4H), 0.83 [app $\mathrm{t}, J=6.3 \mathrm{~Hz}, 6 \mathrm{H}] ;{ }^{13} \mathrm{C}$ NMR $\left(50 \mathrm{MHz}, \mathrm{d}_{6}-\mathrm{DMSO}\right) \delta 176.4\left(\mathrm{~d},{ }^{3} J_{\mathrm{PC}}=7.8 \mathrm{~Hz}\right), 156.4\left(\mathrm{~d},{ }^{3} J_{\mathrm{PC}}\right.$ $=4.1 \mathrm{~Hz}), 137.2,135.9,129.2,128.4,128.1,127.9,127.6,125.7,65.6,49.9\left(\mathrm{~d},{ }^{1} J_{\mathrm{PC}}=104 \mathrm{~Hz}\right)$, $42.6\left(\mathrm{~d}, J_{\mathrm{PC}}=8.9 \mathrm{~Hz}\right), 36.8\left(\mathrm{~d}, J_{\mathrm{PC}}=4.0 \mathrm{~Hz}\right), 29.0\left(\mathrm{~d}, J_{\mathrm{PC}}=12 \mathrm{~Hz}\right), 28.9\left(\mathrm{~d},{ }^{1} J_{\mathrm{PC}}=89 \mathrm{~Hz}\right), 25.6$, 23.0, $21.8 ;{ }^{31} \mathrm{P}$ NMR $\left(81 \mathrm{MHz}, \mathrm{d}_{6}\right.$-DMSO $+3 \%$ TFA) $\delta 46.0$; HRMS (m/z): $[\mathrm{M}+\mathrm{H}]^{+}$calcd. for $\mathrm{C}_{24} \mathrm{H}_{33} \mathrm{NO}_{6} \mathrm{PS}^{+}, 494.1761$ found, 494.1760 .

3-\{[(R)-1-(Benzyloxycarbonylamino)-4-(phenylthio)butyl](hydroxy)phosphoryl\}-2-methylpropanoic acid (5q): Phosphinic acid $\mathbf{1 f}(937 \mathrm{mg}, 2.47 \mathrm{mmol})$ and methacrylic acid (3b) $(255 \mathrm{mg}$, $2.97 \mathrm{mmol}$ ) were subjected to the general HMDS-mediated P-C bond-forming reaction protocol. After the addition of $\mathrm{HCl} 2 \mathrm{M}$ and AcOEt, the two phases were separated and the organic layer was dried with $\mathrm{Na}_{2} \mathrm{SO}_{4}$ and evaporated in vacuo. The residue was solidified on standing, treated with $\mathrm{Et}_{2} \mathrm{O}$ and filtrated. Compound $\mathbf{5 q}$ (d.r. 1:1) was isolated as a white solid in 92\% yield (1.06 g, $2.27 \mathrm{mmol})$. TLC $R_{\mathrm{f}}\left(\mathrm{CHCl}_{3} / \mathrm{MeOH} / \mathrm{AcOH}: 7 / 2 / 1\right)=0.61$. ${ }^{1} \mathrm{H}$ NMR $\left(400 \mathrm{MHz}, \mathrm{d}_{6}\right.$-DMSO) $\delta 7.50 \& 7.49(2 \times \mathrm{d}, J=9.6 \mathrm{~Hz}, 1 \mathrm{H}), 7.26 \& 7.21-7.13(\mathrm{~m}$, $10 \mathrm{H}), 5.07(\mathrm{~d}, J=13 \mathrm{~Hz}, 1 \mathrm{H}), 5.02(\mathrm{dd}, J=13,2 \mathrm{~Hz}, 1 \mathrm{H}), 3.70-3.57(\mathrm{~m}, 1 \mathrm{H}), 3.03-2.85(\mathrm{~m}$, $2 \mathrm{H}), 2.73-2.58(\mathrm{~m}, 1 \mathrm{H}), 2.09-1.97(\mathrm{~m}, 1 \mathrm{H}), 1.94-1.81(\mathrm{~m}, 1 \mathrm{H}), 1.76-1.48(\mathrm{~m}, 4 \mathrm{H}), 1.17$ \& 1.16 $(2 \times \mathrm{d}, J=7.1 \mathrm{~Hz}, 3 \mathrm{H}), 5.07(\mathrm{~d}, J=13 \mathrm{~Hz}, 1 \mathrm{H}) ;{ }^{13} \mathrm{C}$ NMR $\left(101 \mathrm{MHz}, \mathrm{d}_{6}\right.$-DMSO) $\delta 176.7$, $176.63,176.56,156.3\left(\mathrm{~d},{ }^{3} J_{\mathrm{PC}}=4.5 \mathrm{~Hz}\right), 137.1,136.3,129.0,128.3,127.8,127.7,127.5,127.4$, $125.5,65.5,50.3\left(\mathrm{~d},{ }^{3} J_{\mathrm{PC}}=104 \mathrm{~Hz}\right), 50.0\left(\mathrm{~d},{ }^{3} J_{\mathrm{PC}}=105 \mathrm{~Hz}\right), 33.2,33.1\left(\mathrm{~d}, J_{\mathrm{PC}}=3.4 \mathrm{~Hz}\right), 31.4$, $29.5\left(\mathrm{~d},{ }^{1} J_{\mathrm{PC}}=89 \mathrm{~Hz}\right), 29.3\left(\mathrm{~d},{ }^{1} J_{\mathrm{PC}}=89 \mathrm{~Hz}\right), 26.3,25.5,25.4,18.8\left(\mathrm{~d}, J_{\mathrm{PC}}=7.4 \mathrm{~Hz}\right), 18.5(\mathrm{~d}$, $\left.J_{\mathrm{PC}}=5.8 \mathrm{~Hz}\right) .{ }^{31} \mathrm{P}$ NMR $\left(162 \mathrm{MHz}, \mathrm{d}_{6}\right.$-DMSO) $\delta 45.1 ; \mathrm{HRMS}(\mathrm{m} / \mathrm{z}):[\mathrm{M}+\mathrm{Na}]^{+}$calcd. for $\mathrm{C}_{22} \mathrm{H}_{28} \mathrm{NNaO}_{6} \mathrm{P}^{+}, 456.1551$ found, 456.1556 .

$\left(\left[1,1^{\prime}: 3^{\prime}, 1^{\prime \prime}\right.\right.$-Terphenyl]-5'-yl)-2-[(\{(R)-1-[(tert-butoxycarbonyl)amino]-3-phenylpropyl\}(hydro $\left.x y\right)$ ph osphoryl)methyl]propanoic acid (5r): Phosphinic acid $1 \mathbf{d}(100 \mathrm{mg}, 0.33 \mathrm{mmol})$ and acrylic acid 31 [20] (126 mg, $0.40 \mathrm{mmol}$ ) were subjected to the general HMDS-mediated P-C bondforming reaction protocol. After the addition of $\mathrm{HCl} 2 \mathrm{M}$ and AcOEt, the two phases were separated and the organic layer was dried with $\mathrm{Na}_{2} \mathrm{SO}_{4}$ and evaporated in vacuo. The crude product was purified by silica gel column chromatography using $\mathrm{CHCl}_{3} / \mathrm{MeOH} / \mathrm{AcOH}$ 7:0.01:0:01 $\rightarrow$ 7:0.4:0:4 as eluent solvent system. Compound 5r (d.r. 2:1) was isolated as a white foam in 92\% yield (193 mg, $2.27 \mathrm{mmol})$. TLC $\mathrm{R}_{\mathrm{f}}\left(\mathrm{CHCl}_{3} / \mathrm{MeOH} / \mathrm{AcOH}: 7 / 0.5 / 0.5\right)$ $=0.43 .{ }^{1} \mathrm{H}$ NMR $\left(200 \mathrm{MHz}, \mathrm{d}_{6}-\mathrm{DMSO}\right) \delta 7.83-7.06(\mathrm{~m}, 18 \mathrm{H}), 3.73-3.47(\mathrm{~m}, 1 \mathrm{H}), 3.20-2.92$ $(\mathrm{m}, 3 \mathrm{H}), 2.80-2.58(\mathrm{~m}, 1 \mathrm{H}), 2.58-2.37\left(\mathrm{~m}, 1 \mathrm{H}\right.$, overlapped by $\mathrm{d}_{6}$-DMSO), $2.08-1.58(\mathrm{~m}, 4 \mathrm{H})$, $1.34 \& 1.41(2 \times \mathrm{s}, 9 \mathrm{H}) ;{ }^{13} \mathrm{C}$ NMR $\left(50 \mathrm{MHz}, \mathrm{d}_{6}\right.$-DMSO) $\delta 175.6,175.5,175.4,155.9,155.82$, $155.76,141.5,141.4,141.0,140.4,140.3,140.2,129.0,128.8,128.6,128.4,127.7,127.1,126.9$, $125.9,123.5,78.4,78.3,49.5\left(\mathrm{~d},{ }^{1} J_{\mathrm{PC}}=107 \mathrm{~Hz}\right), 49.1\left(\mathrm{~d},{ }^{1} J_{\mathrm{PC}}=106 \mathrm{~Hz}\right), 31.9,31.7,29.2,29.0$, 28.3, 28.0, 26.5; ${ }^{31} \mathrm{P}$ NMR (81 MHz, $\mathrm{d}_{6}$-DMSO) $\delta$ 47.0, 46.7; HRMS (m/z): $[\mathrm{M}+\mathrm{H}]^{+}$calcd. for $\mathrm{C}_{36} \mathrm{H}_{41} \mathrm{NO}_{6} \mathrm{P}^{+}, 614.2666$ found, 614.2666.

\section{Conclusions}

In this work, a useful method for the synthesis of phosphinic dipeptides of type $\mathbf{5}$ is presented. These compounds are valuable synthetic intermediates in the field of phosphinic peptides and protocols that facilitate their preparation are of great interest, especially when 
large quantities are required for medicinal chemistry purposes. Based on the observation that acrylic acids are neglected electrophile alternatives for the key P-Michael addition between $\alpha$-aminophosphinic acids and acrylic acceptors, we propose that an unreactive mixture of an aminophosphinic and an acrylic acid can be activated under silylating conditions by acting simultaneously on both reactants, to afford in a single step the target compounds of type 5. A great advantage of the presented method is that in several cases, bioactive $(R, S)$-isomers of type 5 can be directly isolated from the crude products by means of selective crystallization. This tendency is well-described in the literature and has been used in the past for the synthesis of several dipeptides of type 5; for example, $5 \mathrm{~h}$ and $5 \mathrm{~m}$, which are also described in this report and were found spectroscopically identical to previously reported compounds [20,23]. In particular, compounds $5 \mathbf{h}$ [20] and 5m [23] have been converted to tripeptides that were stereochemically characterized either by NMR [23] or by X-ray crystallographic analysis of their complexes with Zn-metalloproteases [38,39]. Taking together the availability of starting acids and the practicality of the proposed P-C bond-forming reaction, we believe that the reported protocol will greatly contribute to the simplification of synthetic procedures targeting this important class of compounds.

Supplementary Materials: Synthetic protocol and characterization for compounds $3 \mathbf{a}$ and $\mathbf{3} \mathbf{c}-\mathbf{k}$. Copies of ${ }^{1} \mathrm{H},{ }^{13} \mathrm{C}$ and ${ }^{31} \mathrm{P}$ NMR spectra for compounds $3 \mathbf{a}, 3 \mathbf{c}-\mathbf{k}$ and $\mathbf{5 a}-\mathbf{r}$.

Author Contributions: Conceptualization, D.G.; methodology, P.K., K.V., A.L., N.S., K.P., A.Z. and E.K.; analysis and characterization, P.K., K.V., A.L., N.S., K.P., L.D., A.Z. and E.K.; supervision, D.G.; resources, L.D.; writing-original draft preparation, D.G.; writing-review and editing, D.G., P.K., K.V., A.L., N.S., K.P., L.D., A.Z. and E.K. All authors have read and agreed to the published version of the manuscript.

Funding: This research received no external funding.

Institutional Review Board Statement: Not applicable.

Informed Consent Statement: Not applicable.

Data Availability Statement: Not applicable.

Acknowledgments: D.G., P.K. and A.L. would like to acknowledge support by the State Scholarships Foundation (IKY) for P.K. under the "Granting Scholarships for Postgraduate Studies of the secondcycle" action of the operational programme "Development of Human Resources, Education and Lifelong Learning" co-funded by the European Social Fund (ESF) and National Resources (NSRF 2014-2020). D.G. would like to acknowledge support by funds from the Special Account for Research Grants of NKUA (Account No: 10504 \& 16672 \& 17454).

Conflicts of Interest: The authors declare no conflict of interest.

Sample Availability: Samples of the compounds are not available from the authors.

\section{Abbreviations}

HMDS 1,1,1,3,3,3-hexamethyldisilazane

BSA N,O-bis(trimethylsilyl)acetamide

TMSCl chlorotrimethylsilane

\section{References}

1. Talma, M.; Maślanka, M.; Mucha, A. Recent developments in the synthesis and applications of phosphinic peptide analogs. Bioorg. Med. Chem. Lett. 2019, 29, 1031-1042. [CrossRef] [PubMed]

2. Abdou, M.M.; O’Neill, P.M.; Amigues, E.; Matziari, M. Phosphinic acids: Current status and potential for drug discovery. Drug Discov. Today 2018, 24, 916-929. [CrossRef]

3. Georgiadis, D.; Dive, V. Phosphinic Peptides as Potent Inhibitors of Zinc-Metalloproteases. Top. Curr. Chem. 2015, 360, 1-38. [CrossRef] [PubMed]

4. Mucha, A. Synthesis and modifications of phosphinic dipeptide analogues. Molecules 2012, 17, 13530-13568. [CrossRef]

5. Mucha, A.; Kafarski, P.; Berlicki, L. Remarkable potential of the alpha-aminophosphonate/phosphinate structural motif in medicinal chemistry. J. Med. Chem. 2011, 54, 5955-5980. [CrossRef] 
6. Dive, V.; Georgiadis, D.; Matziari, M.; Makaritis, A.; Beau, F.; Cuniasse, P.; Yiotakis, A. Phosphinic peptides as zinc metalloproteinase inhibitors. Cell. Mol. Life Sci. 2004, 61, 2010-2019. [CrossRef] [PubMed]

7. Xu, J. Synthetic Methods of Phosphonopeptides. Molecules 2020, 25, 5894. [CrossRef] [PubMed]

8. Xu, J. Synthesis of Phosphinopeptides and Phosphinodepsipeptides. Asian J. Org. Chem. 2021, 10, 287-295. [CrossRef]

9. Xu, J. Synthetic strategies of phosphonodepsipeptides. Beilstein J. Org. Chem. 2021, 17, 461-484. [CrossRef]

10. Nury, C.; Czarny, B.; Cassar-Lajeunesse, E.; Georgiadis, D.; Bregant, S.; Dive, V. A Pan Photoaffinity Probe for Detecting Active Forms of Matrix Metalloproteinases. ChemBioChem 2013, 14, 107-114. [CrossRef]

11. Bordenave, T.; Helle, M.; Beau, F.; Georgiadis, D.; Tepshi, L.; Bernes, M.; Ye, Y.; Levenez, L.; Poquet, E.; Nozach, H.; et al. Synthesis and In Vitro and In Vivo Evaluation of MMP-12 Selective Optical Probes. Bioconjug. Chem. 2016, 27, 2407-2417. [CrossRef] [PubMed]

12. Razavian, M.; Bordenave, T.; Georgiadis, D.; Beau, F.; Zhang, J.; Golestani, R.; Toczek, J.; Jung, J.-J.; Ye, Y.; Kim, H.-Y.; et al. Optical imaging of MMP-12 active form in inflammation and aneurysm. Sci. Rep. 2016, 6, 38345. [CrossRef]

13. Covaleda, G.; Gallego, P.; Vendrell, J.; Georgiadis, D.; Lorenzo, J.; Dive, V.; Aviles, F.X.; Reverter, D.; Devel, L. Synthesis and Structural/Functional Characterization of Selective M14 Metallocarboxypeptidase Inhibitors Based on Phosphinic Pseudopeptide Scaffold: Implications on the Design of Specific Optical Probes. J. Med. Chem. 2019, 62, 1917-1931. [CrossRef] [PubMed]

14. Kaminska, M.; Bruyat, P.; Malgorn, C.; Doladilhe, M.; Cassar-Lajeunesse, E.; Fruchart Gaillard, C.; De Souza, M.; Beau, F.; Thai, R.; Correia, I.; et al. Ligand-Directed Modification of Active Matrix Metalloproteases: Activity-based Probes with no Photolabile Group. Angew. Chem. Int. Ed. 2021, 60, 18272-18279. [CrossRef]

15. Yiotakis, A.; Georgiadis, D.; Matziari, M.; Makaritis, A.; Dive, V. Phosphinic peptides: Synthetic approaches and biochemical evaluation as Zn-metalloprotease inhibitors. Curr. Org. Chem. 2004, 8, 1135-1158. [CrossRef]

16. Kokkala, P.; Rajeshkumar, T.; Mpakali, A.; Stratikos, E.; Vogiatzis, K.D.; Georgiadis, D. A Carbodiimide-Mediated P-C BondForming Reaction: Mild Amidoalkylation of P-Nucleophiles by Boc-Aminals. Org. Lett. 2021, 23, 1726-1730. [CrossRef] [PubMed]

17. Viveros-Ceballos, J.L.; Ordóñez, M.; Sayago, F.J.; Cativiela, C. Stereoselective Synthesis of $\alpha$-Amino-C-phosphinic Acids and Derivatives. Molecules 2016, 21, 1141. [CrossRef]

18. Yao, Q.; Yuan, C. Enantioselective synthesis of H-phosphinic acids bearing natural amino acid residues. J. Org. Chem. 2013, 78, 6962-6974. [CrossRef]

19. Baylis, E.K.; Campbell, C.D.; Dingwall, J.G. 1-Aminoalkylphosphonous acids. Part 1. Isosteres of the protein amino acids. J. Chem. Soc. Perkin Trans. 1 1984, 2845-2853. [CrossRef]

20. Kokkala, P.; Mpakali, A.; Mauvais, F.X.; Papakyriakou, A.; Daskalaki, I.; Petropoulou, I.; Kavvalou, S.; Papathanasopoulou, M.; Agrotis, S.; Fonsou, T.M.; et al. Optimization and Structure-Activity Relationships of Phosphinic Pseudotripeptide Inhibitors of Aminopeptidases That Generate Antigenic Peptides. J. Med. Chem. 2016, 59, 9107-9123. [CrossRef] [PubMed]

21. Vassiliou, S.; Weglarz-Tomczak, E.; Berlicki, L.; Pawelczak, M.; Nocek, B.; Mulligan, R.; Joachimiak, A.; Mucha, A. Structureguided, single-point modifications in the phosphinic dipeptide structure yield highly potent and selective inhibitors of neutral aminopeptidases. J. Med. Chem. 2014, 57, 8140-8151. [CrossRef] [PubMed]

22. Grembecka, J.; Mucha, A.; Cierpicki, T.; Kafarski, P. The most potent organophosphorus inhibitors of leucine aminopeptidase Structure-based design, chemistry, and activity. J. Med. Chem. 2003, 46, 2641-2655. [CrossRef] [PubMed]

23. Makaritis, A.; Georgiadis, D.; Dive, V.; Yiotakis, A. Diastereoselective solution and multipin-based combinatorial array synthesis of a novel class of potent phosphinic metalloprotease inhibitors. Chem. Eur. J. 2003, 9, 2079-2094. [CrossRef] [PubMed]

24. Jullien, N.; Makritis, A.; Georgiadis, D.; Beau, F.; Yiotakis, A.; Dive, V. Phosphinic tripeptides as dual angiotensin-converting enzyme C-domain and endothelin-converting enzyme-1 inhibitors. J. Med. Chem. 2010, 53, 208-220. [CrossRef] [PubMed]

25. Mores, A.; Matziari, M.; Beau, F.; Cuniasse, P.; Yiotakis, A.; Dive, V. Development of potent and selective phosphinic peptide inhibitors of angiotensin-converting enzyme 2. J. Med. Chem. 2008, 51, 2216-2226. [CrossRef]

26. Matziari, M.; Bauer, K.; Dive, V.; Yiotakis, A. Synthesis of the phosphinic analogue of thyrotropin releasing hormone. J. Org. Chem. 2008, 73, 8591-8593. [CrossRef]

27. Matralis, A.N.; Xanthopoulos, D.; Huot, G.; Lopes-Paciencia, S.; Cole, C.; de Vries, H.; Ferbeyre, G.; Tsantrizos, Y.S. Molecular tools that block maturation of the nuclear lamin A and decelerate cancer cell migration. Bioorg. Med. Chem. 2018, 26, 5547-5554. [CrossRef]

28. Skinner-Adams, T.S.; Lowther, J.; Teuscher, F.; Stack, C.M.; Grembecka, J.; Mucha, A.; Kafarski, P.; Trenholme, K.R.; Dalton, J.P.; Gardiner, D.L. Identification of phosphinate dipeptide analog inhibitors directed against the Plasmodium falciparum M17 leucine aminopeptidase as lead antimalarial compounds. J. Med. Chem. 2007, 50, 6024-6031. [CrossRef]

29. Yiotakis, A.; Vassiliou, S.; Jiráček, J.; Dive, V. Protection of the hydroxyphosphinyl function of phosphinic dipeptides by adamantyl Application to the solid-phase synthesis of phosphinic peptides. J. Org. Chem. 1996, 61, 6601-6605. [CrossRef]

30. Thottathil, J.K.; Ryono, D.E.; Przybyla, C.A.; Moniot, J.L.; Neubeck, R. Preparation of phosphinic acids: Michael additions of phosphonous acids/esters to conjugated systems. Tetrahedron Lett. 1984, 25, 4741-4744. [CrossRef]

31. Chen, H.; Noble, F.; Roques, B.P.; Fournié-Zaluski, M.C. Long lasting antinociceptive properties of enkephalin degrading enzyme (NEP and APN) inhibitor prodrugs. J. Med. Chem. 2001, 44, 3523-3530. [CrossRef] [PubMed] 
32. Raguin, O.; Fournié-Zaluski, M.C.; Romieu, A.; Pèlegrin, A.; Chatelet, F.; Pélaprat, D.; Barbet, J.; Roques, B.P.; Gruaz-Guyon, A. A labeled neutral endopeptidase inhibitor as a potential tool for tumor diagnosis and prognosis. Angew. Chem. Int. Ed. 2005, 44, 4058-4061. [CrossRef]

33. Selvam, C.; Oueslati, N.; Lemasson, I.A.; Brabet, I.; Rigault, D.; Courtiol, T.; Cesarini, S.; Triballeau, N.; Bertrand, H.O.; Goudet, C.; et al. A virtual screening hit reveals new possibilities for developing group III metabotropic glutamate receptor agonists. J. Med. Chem. 2010, 53, 2797-2813. [CrossRef] [PubMed]

34. Chen, H.; Noble, F.; Mothé, A.; Meudal, H.; Coric, P.; Danascimento, S.; Roques, B.P.; George, P.; Fournié-Zaluski, M.C. Phosphinic derivatives as new dual enkephalin-degrading enzyme inhibitors: Synthesis, biological properties, and antinociceptive activities. J. Med. Chem. 2000, 43, 1398-1408. [CrossRef] [PubMed]

35. Goh, K.K.K.; Kim, S.; Zard, S.Z. Free-Radical Variant for the Synthesis of Functionalized 1,5-Diketones. Org. Lett. 2013, 15, 4818-4821. [CrossRef]

36. Gottlieb, H.E.; Kotlyar, V.; Nudelman, A. NMR Chemical Shifts of Common Laboratory Solvents as Trace Impurities. J. Org. Chem. 1997, 62, 7512-7515. [CrossRef]

37. Smith, A.B.; Ducry, L.; Corbett, R.M.; Hirschmann, R. Intramolecular Hydrogen-Bond Participation in Phosphonylammonium Salt Formation. Org. Lett. 2000, 2, 3887-3890. [CrossRef] [PubMed]

38. Maben, Z.; Arya, R.; Georgiadis, D.; Stratikos, E.; Stern, L.J. Conformational dynamics linked to domain closure and substrate binding explain the ERAP1 allosteric regulation mechanism. Nat. Commun. 2021, 12, 5302. [CrossRef]

39. Zervoudi, E.; Saridakis, E.; Birtley, J.R.; Seregin, S.S.; Reeves, E.; Kokkala, P.; Aldhamen, Y.A.; Amalfitano, A.; Mavridis, I.M.; James, E.; et al. Rationally designed inhibitor targeting antigentrimming aminopeptidases enhances antigen presentation and cytotoxic T-cell responses. Proc. Natl. Acad. Sci. USA 2013, 110, 19890-19895. [CrossRef] [PubMed] 